\title{
Investigation of Large Scale Motions in Zero and Adverse Pressure Gradient Turbulent Boundary Layers Using High-Spatial-Resolution PIV
}

\author{
Muhammad Shehzad ${ }^{\mathrm{a}, *}$, Bihai Sun ${ }^{\mathrm{a}}$, Daniel Jovic ${ }^{\mathrm{a}}$, Yasar Ostovan ${ }^{\mathrm{b}}$, Christophe Cuvier ${ }^{\mathrm{b}}$, \\ Jean-Marc Foucaut ${ }^{\mathrm{b}}$, Christian Willert ${ }^{\mathrm{c}}$, Callum Atkinson ${ }^{\mathrm{a}}$, Julio Soria ${ }^{\mathrm{a}}$ \\ ${ }^{a}$ Laboratory for Turbulence Research in Aerospace 83 Combustion (LTRAC), Department of Mechanical and \\ Aerospace Engineering, Monash University, Clayton, 3800, Victoria, Australia \\ ${ }^{b}$ Univ. Lille, CNRS, ONERA, Arts et Metiers Institute of Technology, Centrale Lille, UMR 9014 - LMFL - \\ Laboratoire de Mécanique des Fluides de Lille - Kampé de Fériet, Lille, F-59000, France \\ ${ }^{c}$ Institute of Propulsion Technology, German Aerospace Center (DLR), Cologne, Germany
}

\begin{abstract}
High-spatial-resolution (HSR) two-component, two-dimensional particle-image-velocimetry (2C-2D PIV) measurements of a zero-pressure-gradient (ZPG) turbulent boundary layer (TBL) and an adverse-pressure-gradient (APG)-TBL were taken in the Laboratoire de Mécanique des Fluides de Lille (LMFL) High Reynolds number Boundary Layer Wind Tunnel. The ZPG-TBL has a momentum-thickness based Reynolds number $R e_{\delta_{2}}=\delta_{2} U_{e} / \nu=7,750$ (where $\delta_{2}$ is the momentum thickness and $U_{e}$ is the edge velocity), while the APG-TBL has a $R e_{\delta_{2}}=16,240$ and a Clauser's pressure gradient parameter $\beta=\delta_{1} P_{x} / \tau_{w}=2.27$ (where $\delta_{1}$ is the displacement thickness, $P_{x}$ is the pressure gradient in streamwise direction and $\tau_{w}$ is the wall shear stress). After analysing the single-exposed PIV image data using a multigrid/multipass digital PIV with in-house software, proper orthogonal decomposition (POD) was performed on the data to separate flow-fields into large- and small-scale motions (LSMs and SSMs), with the LSMs further categorized into high- and low-momentum events. The LSMs are energized in the outer-layer and this phenomenon becomes stronger in the presence of an adverse-pressure-gradient. Profiles of the conditionally averaged Reynolds stresses show that the high-momentum events contribute more to the Reynolds stresses than the low-momentum between wall to the end of the log-layer and the opposite is the case in the wake region. The cross-over point of the profiles of the Reynolds stresses from the highand low-momentum LSMs always has a higher value than the corresponding Reynolds stress from the original ensemble at the same wall-normal location. This difference is up to $80 \%$ in Reynolds streamwise and shear stresses and up to $15 \%$ in the Reynolds wall-normal stresses. Furthermore, the cross-over point in the APG-TBL moves further from the wall than in the ZPG-TBL. By removing the velocity fields with LSMs which contribute significantly to the most energetic POD mode, the estimate of the Reynolds streamwise stress and Reynolds shear stress from the remaining fluctuating velocity fields is reduced by up to $42 \%$ in the ZPG-TBL. The reduction effect is observed to be even larger (up to 50\%) in the APG-TBL. However, the removal of these LSMs has a minimal effect on the Reynolds wall-normal stress
\end{abstract}

\footnotetext{
${ }^{*}$ Corresponding author. Email Address: muhammad.shehzad@monash.edu
} 
in both the ZPG and the APG cases.

Keywords: High Spatial Resolution, PIV, Large Scale Motions, Turbulent Boundary Layer, Adverse Pressure Gradient, Zero Pressure Gradient

\section{Introduction}

The identification and characterization of coherent structures in turbulent flows has been an active area of research for decades. In wall-bounded flows, LSMs are defined as coherent patterns that dominate the log-layer and are characterized as alternating regions of highand low-momentum (Kline et al., 1967; Wark and Nagib, 1991; Ganapathisubramani et al., 2005; Del Alamo and Jiménez, 2003; Hambleton et al., 2006). Large-scale coherent motions in the log-law layers are often referred to as superstructures in turbulent boundary layers (Bross et al., 2021, 2019; Mejia-Alvarez et al., 2014; Barros and Christensen, 2019) and very large scale motions (VLSMs) in turbulent pipe flows (Guala et al., 2006; Hellström et al., 2011; Hellstrom et al., 2015) and turbulent channel flows (Lee et al., 2014; Liu et al., 2001; de Giovanetti et al., 2017) in the literature. These structures also greatly influence the nearwall region as they superimpose onto the near-wall SSMs and hence, leave their footprints at the wall (Hutchins and Marusic, 2007a). Furthermore, LSMs in the log-layer cause an amplitude modulation of the SSMs in the near-wall region (Mathis et al., 2009; Harun et al., 2013; Hutchins and Marusic, 2007b). These regions of LSMs are elongated up to the order of $20 \delta$ in the streamwise direction (Hutchins and Marusic, 2007a), where $\delta$ is the boundary layer thickness. They are distinguished from the very thin low-speed streaks in the buffer layer at high Reynolds numbers due to their much larger wall-normal extent (Liu et al., 2001). A slightly inclined and elongated streamwise velocity correlation function implies that the LSMs are significant contributors to the streamwise turbulent kinetic energy (TKE) as observed by both Grant (1958) and Townsend (1958).

While there have been a number of studies dealing with the statistical nature of TBLs under the influence of a pressure gradient (see Nagano et al. (1993); Spalart and Watmuff (1993); Skaare and Krogstad (1994); Krogstad and Skåre (1995); Fernholz and Warnack (1998); Na and Moin (1998); Bourassa and Thomas (2009); Kitsios et al. (2017); Cuvier et al. (2017); Sekimoto et al. (2019); Senthil et al. (2020) among others), literature reveals that only a few characterize the coherent structures (Lian, 1990; Zhou and Lu, 1997; Houra et al., 2000; Lee and Sung, 2009; Drozdz and Elsner, 2011; Kitsios et al., 2017; Sekimoto et al., 2017; Senthil et al., 2020) and fewer characterize the LSMs (Harun et al., 2013; Hain et al., 2016; Bross et al., 2019; Eich and Kähler, 2020). Using spectral analysis, Harun et al. (2013) studied the effect of pressure gradients on the LSMs in a ZPG-TBL at a $R e_{\delta_{2}}=8,160$ and in an APG-TBL at a $R e_{\delta_{2}}=12,030$ and a $\beta=1.74$. They observed that the large scales are energized in the entire APG-TBL and that the amplitude modulation of near-wall small scales by the LSMs increases with an increasing pressure gradient.

Bross et al. (2019) studied the interaction of coherent structures in the near-wall region of an APG-TBL using high-resolution time-resolved 2D and 3D particle tracking velocimetry (PTV). They reported that the high-momentum LSMs in the buffer- and log-layer region were associated with positive fluctuating wall-shear stress $\left(+\tau_{w}^{\prime}\right)$ and the low-momentum LSMs were associated with negative fluctuating wall-shear stress $\left(-\tau_{w}^{\prime}\right)$. Thus, the LSMs in the loglayer significantly manipulate the wall-shear stress, $\tau_{w}^{\prime}$. They also presented a three-layered 
model of uniform-momentum-zones (UMZs) in a TBL, of which the first zone is associated with the viscous sublayer, the second is formed by the high- and low-speed streaks in the buffer-layer and the third is generated by the high- and low-momentum LSMs in the loglayer. When these low- and high-momentum LSMs coincide with ejections or sweeps (i.e. Q2 and Q4 events according to the quadrant analysis of Wallace et al. (1972)), the number of UMZs strongly increases or decreases, respectively (Paakkari et al., 2018). Hence, Bross et al. (2019) conclude that the momentum of log-layer LSMs and their footprint on the wall are related to the number of UMZs in the instantaneous velocity fields.

Proper Orthogonal Decomposition (POD) has been used as a tool to study the coherent structures, including LSMs since its first application in fluid dynamics by Lumley (1967). It is a generalization of the conventionally used Fourier power spectral analysis and is used to investigate the TKE distribution as a function of scale in a TBL flow that is inhomogeneous in the streamwise direction (Liu et al., 2001). POD has been used to study the randomly distributed counter-rotating eddies as LSMs in a turbulent pipe flow by Bakewell Jr and Lumley (1967) at a $R e_{D}=U_{b} D / \nu=8,700$, where $U_{b}$ is the bulk velocity and $D$ is the pipe diameter. Liu et al. (2001) used POD to evaluate the scales contributing towards the events that produce TKE and Reynolds shear stress from the 2D data of two different channel flows at a $R e_{h}=U_{b} h / \nu=5,378$ and 29,935 (where $h$ represents the channel half-height) and concluded that the LSMs contain a large fraction of the Reynolds streamwise stress component and a small fraction of Reynolds wall-normal stress component. Wu (2014) studied the LSMs in a TBL with a zero pressure gradient (ZPG) at two different configurations, $R e_{\delta_{2}}=8200$ and 12000, and performed POD to establish a connection between the first two dominant POD modes and the instantaneous large scale structures. The authors concluded that the Reynolds streamwise stress, Reynolds shear stress and spatial velocity correlation functions are reduced without the LSMs that significantly contribute to the dominant first POD mode.

By performing POD on a dataset, the LSMs are characterized as structures whose contribution to the dominant spatial mode is above a threshold. The classification of LSMs into high- and low-momentum events is based on the nature of the first POD mode. Conditional averaging of velocity fields based on this classification leads to the analysis of the contribution of the extreme events to the turbulent statistics. Guemes et al. (2019) studied the LSMs in a ZPG-TBL by using POD to investigate the effect of high- and low-momentum events on turbulent statistics. They observed that the high-momentum events have a larger influence on the mean flow and Reynolds stresses near the wall when compared to the low-momentum events, and this effect decreases with an increasing distance from the wall. Conversely, the low-momentum events had a weaker contribution to the inner peak and a stronger contribution to the outer peak in Reynolds streamwise stress profile. To the best of authors' knowledge, the effect of the high and low-momentum events on the turbulent statistics in TBL under the influence of an adverse pressure gradient has not yet been investigated.

Earlier studies on the LSMs in wall-bounded flows were carried out using either singlepoint measurements or low-spatial-resolution 2C-2D PIV measurements. To investigate the effect of high- and low-momentum events near the wall, HSR measurements are of immense importance. In the present paper, HSR 2C-2D PIV measurements have been used to investigate the LSMs and the effect of high- and low-momentum events on the turbulent statistics in both the ZPG- and the APG-TBLs. This paper is organized in the following manner: The methodology of the POD on the instantaneous fluctuating flow field and classification 
of LSMs into high- and low-momentum events is presented in Section 2. Section 3 describes the experimental details of the HSR 2C-2D PIV measurements. The first- and second-order statistics, conditionally averaged statistics and sensitivity analysis of different threshold limits along with discussion are presented in Section 4. Lastly, the conclusions are presented in Section 5 .

Throughout this paper, we take $x, y$ and $z$ as the streamwise, wall-normal and spanwise directions, respectively. The instantaneous, mean and fluctuating velocities in the $x$ directions are referred to as $u, U$ and $u^{\prime}$, respectively. Accordingly, the velocities in $y$ are represented by $v$.

\section{Identification of the Large Scale Motions using POD}

\subsection{Proper Orthogonal Decomposition (POD)}

POD using a snapshot method was introduced by Sirovich (1987), briefly discussed in Taira et al. (2017) and used in this study to extract modes based on optimizing the mean square of the fluctuating velocity. The method is briefly presented below.

Consider a set of velocity fields $\overline{\overline{\boldsymbol{u}^{\prime}}}(x, t)$,

$$
\overline{\overline{\boldsymbol{u}^{\prime}}}(x, t)=\left[\begin{array}{llll}
\boldsymbol{u}^{\prime}\left(x, t_{1}\right) & \boldsymbol{u}^{\prime}\left(x, t_{2}\right) & \ldots & \boldsymbol{u}^{\prime}\left(x, t_{N}\right)
\end{array}\right] \in \mathbb{R}^{M \times N}, \quad M \gg N
$$

where $N$ is the number of snapshots (i.e. velocity fields) and $\mathrm{M}$ is twice the number of grid points in each snapshot and

$$
\boldsymbol{u}^{\prime}(x, t)=\left[\begin{array}{l}
u^{\prime}(x, t) \\
v^{\prime}(x, t)
\end{array}\right]
$$

$\overline{\overline{\boldsymbol{u}^{\prime}}}(x, t)$ can be decomposed by POD in the following manner

$$
\overline{\overline{\boldsymbol{u}^{\prime}}}(x, t)=\sum_{i=1}^{N} \psi_{i}(t) \phi_{i}(x)
$$

where $\phi_{i}(x)$ is the $i$ th spatial mode and $\psi_{i}(t)$ is the set of the corresponding temporal coefficients.

Let $\boldsymbol{X}=\overline{\overline{\boldsymbol{u}^{\prime}}}$. In the regular POD,

$$
\boldsymbol{R} \phi_{i}=\lambda_{i} \phi_{i}, \quad \phi_{i} \in \mathbb{R}^{M}, i=1,2, \ldots, M
$$

where $\lambda_{i}$ represents the eigenvalue of the $i$ th mode and $\boldsymbol{R}$ is the covariance matrix of vector $\boldsymbol{u}^{\prime}(x, t)$ such that

$$
\boldsymbol{R}=\boldsymbol{X} \boldsymbol{X}^{T}, \quad \boldsymbol{R} \in \mathbb{R}^{M \times M}
$$

In snapshot-POD, the matrix $\boldsymbol{X}^{T} \boldsymbol{X}$ is used which is much smaller in size as compared to the regular POD matrix $\boldsymbol{X} \boldsymbol{X}^{T}$ and yet has the same nonzero eigenvalues (Sirovich, 1987). Hence we can write

$$
\boldsymbol{X}^{T} \boldsymbol{X} \psi_{i}=\lambda_{i} \psi_{i}, \quad \psi_{i} \in \mathbb{R}^{N},
$$


With the eigenvector (i.e. vector of temporal coefficients) $\psi_{i}$ and eigenvalue $\lambda_{i}$, the corresponding spatial mode $\phi_{i}$ can be computed as

$$
\phi_{i}=\boldsymbol{X} \psi_{i} \frac{1}{\sqrt{\lambda_{i}}}
$$

This can also be written as

$$
\Phi=\boldsymbol{X} \boldsymbol{\Psi} \Lambda^{-1 / 2}
$$

where the columns of $\boldsymbol{\Phi}$ are the vectors of the spatial modes $\left(\boldsymbol{\Phi}=\left[\begin{array}{lll}\phi_{1} & \phi_{2} \ldots \phi_{N}\end{array}\right] \in \mathbb{R}^{M \times N}\right)$, the columns of $\boldsymbol{\Psi}$ are the vectors of temporal coefficients corresponding to each POD mode $\left(\boldsymbol{\Psi}=\left[\psi_{1} \psi_{2} \ldots \psi_{N}\right] \in \mathbb{R}^{N \times N}\right)$ and $\boldsymbol{\Lambda}$ is the vector of eigenvalues corresponding to each POD mode $\left(\boldsymbol{\Lambda}=\left[\begin{array}{llll}\lambda_{1} & \lambda_{2} & \ldots & \lambda_{N}\end{array}\right] \in \mathbb{R}^{N}\right)$.

The TKE equals to half of the sum of the eigenvalues, i.e.

$$
k=\frac{1}{2} \overline{\boldsymbol{u}^{\prime 2}}=\frac{1}{2} \sum_{i=1}^{N} \lambda_{i}
$$

\subsection{Classification of the Large Scale Motions}

As described in $\mathrm{Wu}$ (2014), the velocity fields dominated by LSMs are identified as the fields for which the magnitude of the temporal coefficients $\psi_{i_{j}}$ are beyond a threshold $K \sigma_{\psi_{i}}$, where $\sigma_{\psi_{i}}$ is the standard deviation of the temporal coefficients corresponding to the $i$ th POD mode, and $K=\{1.0,1.5,2.0\}$ is a threshold factor. As the first POD mode contains the largest contribution of the TKE and the streamwise velocity fluctuations reconstructed from the first mode $\left(u_{\phi_{1}}^{\prime}\right)$ are either purely positive or negative, we take $i=1$ to separate the flow field into the velocity fields with LSMs and SSMs.

$$
\begin{gathered}
F\left(\left|\psi_{i}\right|>K \sigma_{\psi_{i}}\right) \longrightarrow F(L S M), \\
F\left(\left|\psi_{i}\right|<K \sigma_{\psi_{i}}\right) \longrightarrow F(S S M)
\end{gathered}
$$

The LSMs are further classified into the high-momentum $\left(u^{\prime}>0\right)$ and low-momentum $\left(u^{\prime}<0\right)$ events based on the nature of the first POD mode (Wu, 2014; Guemes et al., 2019). If the streamwise component of the first mode is positive, i.e. $u_{\phi_{1}}^{\prime}>0$, the snapshots of the flow field $F$ with their temporal coefficients $\psi_{1_{j}}$ larger than $K \sigma_{\psi_{1}}$ are identified as those with dominant high-momentum events $\left(H^{+}\right)$and those with $\psi_{1}$ smaller than $-K \sigma_{\psi_{1}}$ are identified as those with dominant low-momentum events $\left(L^{+}\right)$.

$$
\begin{gathered}
F\left(\psi_{1}>K \sigma_{\psi_{1}}\right) \longrightarrow H^{+}, \\
F\left(\psi_{1}<-K \sigma_{\psi_{1}}\right) \longrightarrow L^{+}
\end{gathered}
$$

Conversely, if $u_{\phi_{1}}^{\prime}<0$, the snapshots of the flow field $F$ with $\psi_{1}>K \sigma_{\psi_{1}}$ are identified as fields with dominant low-momentum events $\left(L^{-}\right)$, and those with $\psi_{1}<-K \sigma_{\psi_{1}}$ as fields with dominant high-momentum events $\left(H^{-}\right)$

$$
\begin{aligned}
F\left(\psi_{1}>K \sigma_{\psi_{1}}\right) & \longrightarrow L^{-}, \\
F\left(\psi_{1}<-K \sigma_{\psi_{1}}\right) & \longrightarrow H^{-}
\end{aligned}
$$

The + and - superscripts have been adopted to differentiate between the nature of the 
first POD mode and subsequent classification in the two data-sets (i.e. of ZPG- and APGTBLs). The basis of the selection of the optimal value of $K$ is described in section 4.2 and compared with the other values to analyse their effect on the conditionally averaged turbulent statistics in section 4.4 .

\section{Experimental methodology}

\subsection{Facility and Apparatus}

High-spatial-resolution 2C-2D PIV images were taken in the $x-y$ plane in the HighReynolds-Number Boundary Layer Wind Tunnel at LMFL, Lille, France. This facility has a $2 m$-wide, $1 m$-high and 20.6m-long test section. A schematic view of the wind tunnel facility is shown in figure 1 with the three different sections indicated; with a zero-pressure-gradient, with a favourable-pressure-gradient (FPG) and finally with an adverse-pressure-gradient. Previously in the EuHIT experiment (Cuvier et al., 2017), 2C-2D PIV measurements of an FPG-TBL with four sCMOS cameras at four stations and an APG-TBL with 16 sCMOS cameras in a $3.466 \mathrm{~m}$ long continuous field of view (FOV) were obtained to characterize the high Reynolds number FPG- and APG-TBL developing over considerably long regions of the test section before entering the FPG region. The details of the EuHIT experiment are reported in Cuvier et al. (2017). 2C-2D Planar PIV and 3C-3D Stereo-PIV (SPIV) measurements of the ZPG-TBL were taken at $x=3.2 \mathrm{~m}$ and $x=6.8 \mathrm{~m}$ respectively to characterize the inlet conditions of the TBL.

To study the dynamics of the TBL in the near-wall and log regions, HSR 2C-2D PIV measurements of a ZPG-TBL were taken at $x=6.8 \mathrm{~m}$, whereas the measurements of the APG-TBL were taken at $s=5.6 m$, where $s$ is the curvilinear coordinate along the ramp surface with $s=0$ at the beginning of the ramp. The inflow velocity for both measurements is $9 \mathrm{~m} / \mathrm{s}$. A 29 Megapixel Imperx Bobcat B6640 camera was used to record these images. The camera has a CCD sensor with $6576 \times 4384$ pixels and a pixel size of $5.5 \mu m$. The image magnification was $12.684 \mu \mathrm{m} / p x$ for ZPG-TBL and $12.726 \mu \mathrm{m} / p x$ for APG-TBL. The FOV was $(56.190 \times 46.449) \mathrm{mm}$ for the ZPG-TBL and $(47.213 \times 49.656) \mathrm{mm}$ for the APG-TBL. Due to the limited number of high-resolution cameras available, HSR 2C-2D PIV measurements of the whole APG region in the LMFL wind tunnel were not possible. Therefore, APG-TBL measurements were taken only at one station in the APG region. The FOV was illuminated by a dual cavity, frequency-doubled Innolas Nd:YAG laser for ZPG-TBL and BMI laser for APG-TBL with the maximum energy of $150 \mathrm{~mJ}$ and $200 \mathrm{~mJ}$ per pulse at a wavelength of $532 \mathrm{~nm}$. The laser sheet was created by a combination of converging and diverging lenses to reduce the thickness in the out-of-plane direction and expand the laser beam to form a sheet in the in-plane direction. Two cylindrical lenses of focal lengths of $f=-1000 \mathrm{~mm}$ at the output of the laser and $f=+400 \mathrm{~mm}$ at about $400 \mathrm{~mm}$ upstream the FOV were used to create the laser sheet for the ZPG-TBL measurements. For the APG-TBL measurements, a cylindrical lens of $f=-40 \mathrm{~mm}$ and a spherical lens of $f=1500 \mathrm{~mm}$ were used to create the required laser sheet. The thickness of the laser sheet across the field of view was measured to be about $400 \mu m$ for the ZPG-TBL and about $200 \mu m$ for the APG-TBL. The laser sheet for the ZPG-TBL was introduced from underneath the glass-floor of the wind tunnel at $x=6.8 \mathrm{~m}$. For the APG-TBL, the laser sheet was introduced from the bottom at a location downstream of the APG region but mirrored to align its bottom edge coincident with the 


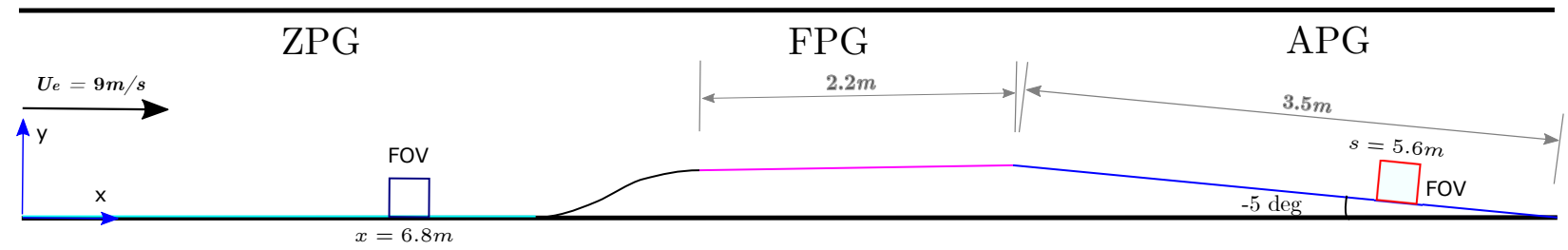

Figure 1: Schematic of the test section in the LML Wind Tunnel. Figure adapted from Cuvier et al. (2017).

Table 1: Boundary layer parameters from EuHIT experiment. Source: Cuvier et al. (2017)

\begin{tabular}{|c|c|c|c|c|c|c|c|}
\hline TBL & $U_{e}(m / s)$ & $\delta(m m)$ & $\delta_{1}(m m)$ & $\delta_{2}(m m)$ & $H$ & $R e_{\delta_{2}}$ & $\beta$ \\
\hline $\mathrm{ZPG}$ & 9.64 & 102 & 16.4 & 12.0 & 1.37 & 7,750 & - \\
\hline $\mathrm{APG}$ & 11.59 & 175 & 33.5 & 21.0 & 1.45 & 16,240 & 2.27 \\
\hline
\end{tabular}

$-5^{\circ}$ inclined plate. The elapsed time between the two laser pulses $\Delta t$ was set to be $30 \mu s$ for both TBLs to achieve an optical maximum particle image displacement of 20px on the sensor. The flow was seeded with droplets of a water-glycol mixture with a mean diameter of around $1 \mu \mathrm{m}$, using a smoke generator. For each TBL, over 10, 000 image pairs were recorded. As the purpose was to take HSR 2C-2D PIV images, the measurements for both TBLs could not capture the whole boundary layer, but instead only a region from the wall up to the beginning of the wake region. The boundary layer parameters for both TBLs which could not be computed from the current HSR 2C-2D PIV data, have been adopted from Cuvier et al. (2017) as presented in table 1.

\subsection{Distortion Correction}

When measuring with large imaging sensors, lens distortions are introduced into the PIV images. These are usually radial distortions which are a minimum at the centre of the image and a maximum in the corners. To correct for these distortions, the PIV images are dewarped using a second-order rational function (R22) (Willert, 1997) given below:

$$
\begin{gathered}
\hat{x}_{i_{j}}=\frac{a_{11} \hat{x}_{o_{j}}+a_{12} \hat{y}_{o_{j}}+a_{13}+a_{14} \hat{x}_{o_{j}}^{2}+a_{15} \hat{x}_{o_{j}} \hat{y}_{o_{j}}+a_{16} \hat{y}_{o_{j}}^{2}}{a_{31} \hat{x}_{o_{j}}+a_{32} \hat{y}_{o_{j}}+a_{33}+a_{34} \hat{x}_{o_{j}}^{2}+a_{35} \hat{x}_{o_{j}} \hat{y}_{o_{j}}+a_{36} \hat{y}_{o_{j}}^{2}}, \\
\hat{y}_{i_{j}}=\frac{a_{21} \hat{x}_{o_{j}}+a_{22} \hat{y}_{o_{j}}+a_{23}+a_{24} \hat{y}_{o_{j}}^{2}+a_{25} \hat{x}_{o_{j}} \hat{y}_{o_{j}}+a_{26} \hat{y}_{o_{j}}^{2}}{a_{31} \hat{x}_{o_{j}}+a_{32} \hat{y}_{o_{j}}+a_{33}+a_{34} \hat{x}_{o_{j}}^{2}+a_{35} \hat{x}_{o_{j}} \hat{y}_{o_{j}}+a_{36} \hat{y}_{o_{j}}^{2}}, \\
a_{33}=1
\end{gathered}
$$

where $\left(\hat{x}_{i_{j}}, \hat{y}_{i_{j}}\right)$ and $\left(\hat{x}_{o_{j}}, \hat{y}_{o_{j}}\right)$ are coordinates of the image points and the object points in the centred and normalized image space $\left\{\hat{P}_{I}\right\}$ and object space $\left\{\hat{P}_{O}\right\}$ respectively. $j=$ $\left[1,2,3, \ldots, N_{m}\right]$ represents a pair of the corresponding image and object points for an individual marker and $N_{m}$ is the total number of markers in the calibration target image.

The locations of all the markers are obtained with a sub-pixel accuracy by cross-correlating the calibration image with a custom marker template and using a peak finding algorithm. This creates the image space, $\overline{P_{I}}$. The approximate magnification $M$ is calculated from the 
average distance between the four markers in the centre of the image space because distortion is minimum there. $M$ is then used to create the object space $\left\{\overline{P_{O}}\right\}$ with its origin coincident with the origin of the image space. The centred and normalized image space and object space are obtained from their original counter parts $\overline{P_{I}}$ and $\overline{P_{O}}$ as follows:

$$
\begin{array}{r}
\left\{\hat{P}_{I}\right\}=\frac{\left\{\overline{P_{I}}\right\}-\overline{P_{i_{c}}}}{x_{o_{\max }}} \\
\left\{\hat{P}_{O}\right\}=\frac{\left\{\overline{P_{O}}\right\}-\overline{P_{o_{c}}}}{x_{o_{\max }}}
\end{array}
$$

where $\overline{P_{i_{c}}}$ and $\overline{P_{o_{c}}}$ are the image and object points at the middle of the image space and object space respectively and $x_{o_{\max }}$ is the largest $x$ coordinate in the object space.

The difference of the centred and normalized object and image points is highlighted in Fig. 2 where the arrows extend from the object points towards their corresponding image points. The maximum difference of the image points and object points are observed to be 6.73px and 5.68px in the ZPG- and APG-TBLs PIV images respectively. As shown in the figure, the distortion in ZPG-TBL images is a combination of a solid body rotation and pincushion type while in APG-TBL, it is mostly the pincushion type distortion.

Complete details of the distortion correction method are given in Sun et al. (2021). The characteristic parameters required to compute the dewarping coefficients

$$
\left[a_{11}, a_{12}, \ldots, a_{16}, a_{21}, a_{22}, \ldots, a_{26}, a_{31}, a_{32}, \ldots, a_{36}\right]
$$

of equation 13 for distortion correction of the current HSR ZPG- and APG-TBLs measurements are listed in table 2.

Table 2: The characteristic parameters to compute the dewarping coefficients of the equation 13.

\begin{tabular}{cccc}
\hline \hline Property & Units & ZPG & APG \\
\hline$\overline{\Delta x}_{o}, \overline{\Delta y}_{o}$ & $(m m)$ & $1 \pm 0.001$ & $1 \pm 0.001$ \\
$M$ & $(p x / m m)$ & 78.839 & 78.580 \\
$\bar{P}_{I_{c}}$ & $(p x)$ & $(2108.7,1697.7)$ & $(1865.1,1860.8)$ \\
$\bar{P}_{O_{c}}$ & $(p x)$ & $(2105.8,1700.6)$ & $(1863.7,1858.0)$ \\
$x_{O_{\max }}$ & $(p x)$ & 4392.1 & 3671.3 \\
$\hat{P}_{I_{\min }}$ & $(p x)$ & $(-0.467,-0.378)$ & $(-0.493,-0.493)$ \\
$\hat{P}_{I_{\max }}$ & $(p x)$ & $(0.521,0.395)$ & $(0.493,0.514)$ \\
$\hat{P}_{O_{\min }}$ & $(p x)$ & $(-0.467,-0.377)$ & $(-0.492,-0.492)$ \\
$\hat{P}_{O_{\max }}$ & $(p x)$ & $(0.521,0.395)$ & $(0.492,0.514)$ \\
\hline
\end{tabular}

\subsection{PIV Analysis}

The PIV images were analysed using a multigrid/multipass digital PIV (Soria, 1996) with in-house software. The parameters of the PIV analysis are given in terms of viscous units in 

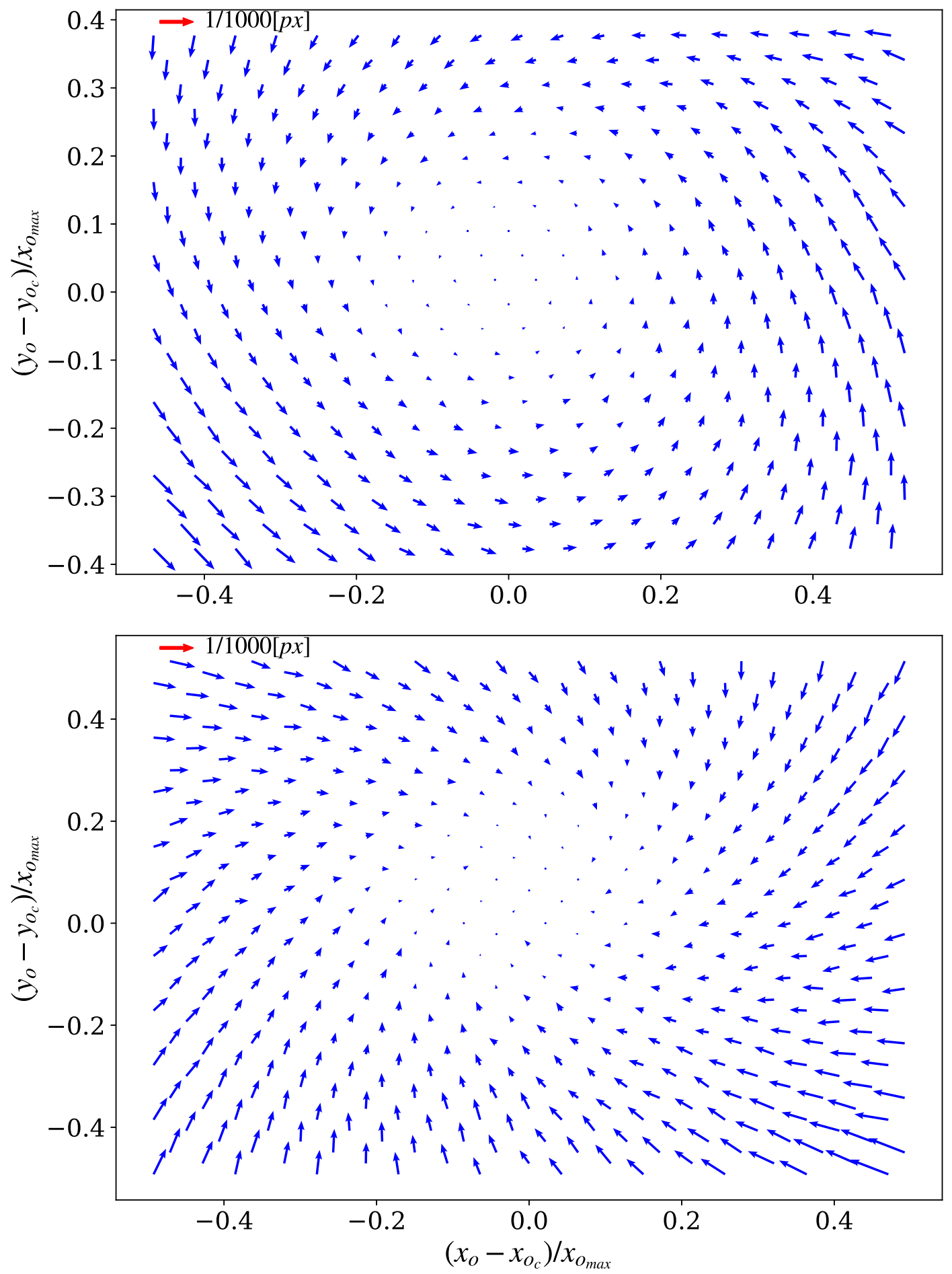

Figure 2: Difference of the centred and normalized object and image points with blue arrows pointing from the object points towards the corresponding image points for the ZPG on top and APG on bottom. The number of arrows (i.e. markers) have been down-sampled to help visualize it better. 
table 3. In order to compare the first and second-order statistics in the outer layer, profiles of the ZPG- and APG-TBLs from the EuHIT experiment measured at the same streamwise stations and their PIV analysis parameters, are presented in section 4.1. These have been adopted from Cuvier et al. (2017). The current measurements are approximately 3 times more resolved in the streamwise direction for both of the TBLs while being about 27 and 8 times more resolved in wall-normal direction for the ZPG- and the APG-TBLs respectively when compared to their EuHIT counterparts from Cuvier et al. (2017). As the current PIV measurements have significantly higher resolution in the wall-normal direction, the wall shear stress $\tau_{w}=\mu\left(\frac{d U}{d y}\right)_{y=0}$ and friction velocity $u_{\tau}=\sqrt{\tau_{w} / \rho}$ for both the TBLs are measured directly from wall-normal gradient of the mean streamwise velocity in the viscous sublayer at every streamwise grid-point. The mean values of the measured friction velocities are 0.35 and 0.33 for ZPG- and APG-TBL. These values are in good agreement with those measured in Cuvier et al. (2017) with a deviation of about $1 \%$.

Table 3: PIV analysis parameters. Source for EuHIT data: Cuvier et al. (2017)

\begin{tabular}{ccccc}
\hline \hline Measurement & ZPG & APG & ZPG(EuHIT) & APG(EuHIT) \\
\hline Inflow Velocity $(m / s)$ & 9 & 9 & 9 & 9 \\
Viscous length scale $l^{+}(\mu m)$ & 42 & 44 & 42 & 44 \\
FOV $\left(l^{+} \times l^{+}\right)$ & $1,492 \times 1,090$ & $1,456 \times 1,115$ & $7,085 \times 4,265$ & $78,847 \times 5801$ \\
Grid spacing $\left(l^{+} \times l^{+}\right)$ & $5 \times 1$ & $5 \times 2$ & $26 \times 39$ & $34 \times 34$ \\
$(p x \times p x)$ & $16 \times 4$ & $16 \times 6$ & $11 \times 11$ & $14 \times 14$ \\
IW size $\left(l^{+} \times l^{+}\right)$ & $19 \times 2$ & $19 \times 7$ & $57 \times 57$ & $58 \times 58$ \\
$(p x \times p x)$ & $64 \times 8$ & $64 \times 24$ & $24 \times 16$ & $24 \times 24$ \\
Number of samples & 10,479 & 10,479 & 10,000 & 30,000 \\
Vector field size & $269 \times 593$ & $224 \times 633$ & $299 \times 180$ & $3,250 \times 238$ \\
Frequency $(H z)$ & 1.5 & 1.5 & 5 & 4 \\
\hline
\end{tabular}

\section{Results and discussion}

\subsection{First- and second-order statistics}

Mean streamwise velocity profiles of ZPG- and APG-TBLs normalized by the viscous units are shown in Fig. 3. The ZPG-TBL has a longer log-law region than that of the APG-TBL. For comparison, the outer-layer mean velocity profiles of the ZPG- and APG-TBLs from the EuHIT experiment have also been included which are consistent with their respective profile from the current HSR measurements. This is expected because of the same facility 
and nearly matching experimental conditions used for the two experiments. Moreover, the near-wall profiles of ZPG-TBL (Willert et al., 2018) and APG-TBL (Cuvier et al., 2017) obtained using time-resolved 2C-2D PIV (TRPIV) have also been included. As the current HSR PIV profiles collapse well with the profiles from the earlier PIV measurements in the respective pressure gradient, this shows sufficient repeatability of the experiments.

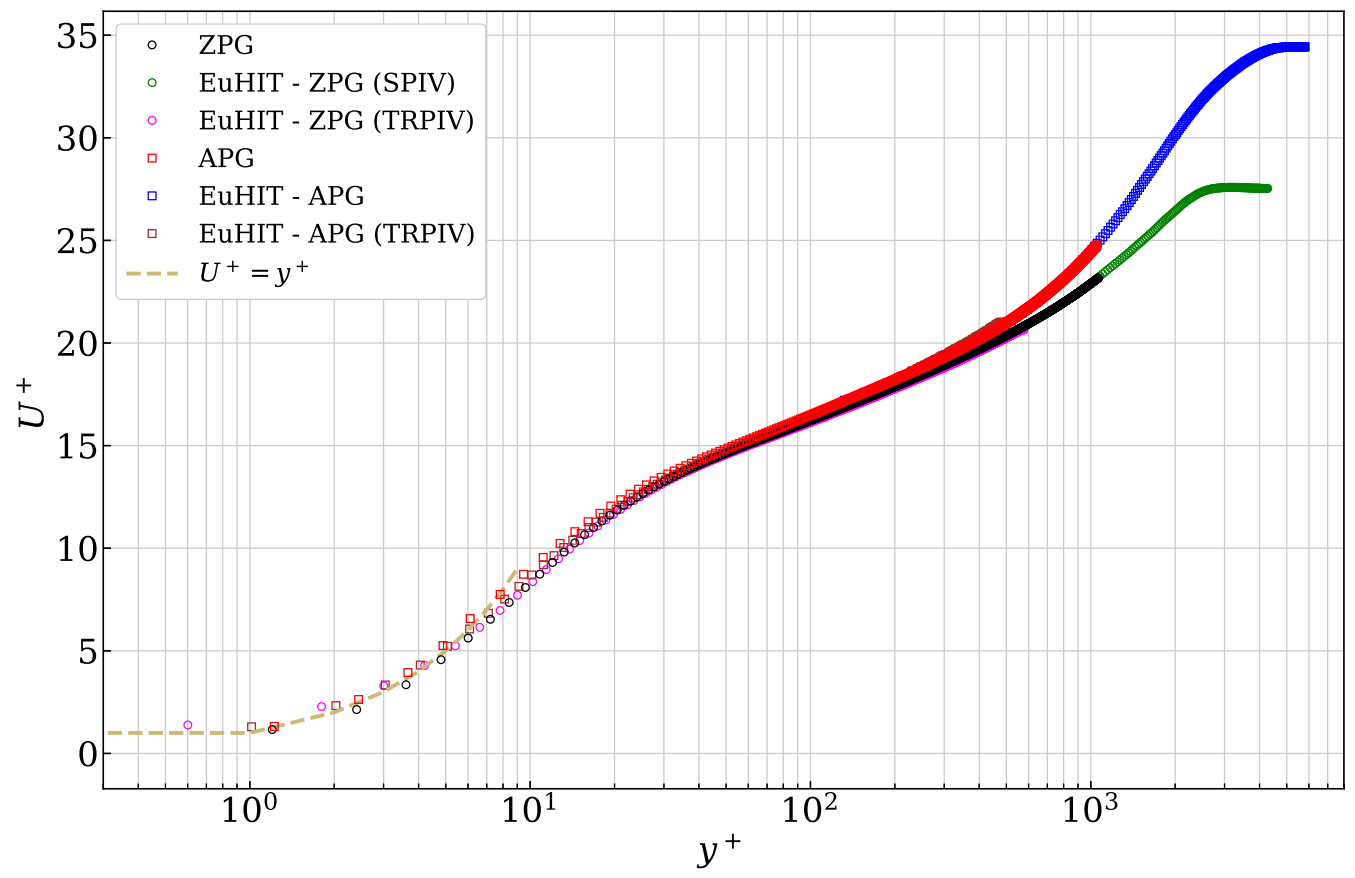

Figure 3: Mean velocity profiles.

Reynolds stress profiles scaled in inner variables and outer variables are shown in Fig. 4. The ZPG-TBL has roughly a $20 \%$ lower inner peak in the Reynolds streamwise stress $\left(\overline{u^{\prime} u^{\prime}}\right)$ at $y^{+} \approx 15$ (where $y^{+}$denotes the wall viscous units) profile when compared to the APG-TBL and has no outer peak, which is consistent for TBLs in this Reynolds number regime. Whereas in the APG-TBL, the outer peak located at $y / \delta \approx 0.2$ (where $\delta$ denotes the boundary layer thickness) is almost as strong as the inner peak. The profile of Reynolds wallnormal stress $\overline{v^{\prime} v^{\prime}}$ has a less pronounced peak at $y / \delta \approx 0.2$ in the ZPG-TBL but is still more than two times weaker than the peak in the APG-TBL, which is also located at $y / \delta \approx 0.2$. Reynolds shear stress $\overline{u^{\prime} v^{\prime}}$ shows a plateau in the region $y^{+} \approx 100-300$ in the ZPG-TBL and an outer peak at $y / \delta \approx 0.2$ in the APG-TBL. This shows that turbulence moves away from the wall towards the outer region when the TBL is under the influence of an APG. Profiles of Reynolds stresses in the outer layer from the EuHIT experiment have also been included for comparison. These profiles deviate by less than $5 \%$ from the HSR measurements in the region $y^{+} \approx 130-500$ which could be the result of their low-spatial-resolution and more averaging due to larger interrogation windows (IWs) used. Above $y / \delta \approx 0.15$, EuHIT profiles are in good agreement with the current HSR measurements. The near-wall profiles of ZPG- and APG-TBL from the TRPIV have also been included. While these profiles are 
in good agreement with the current HSR measurements for Reynolds wall-normal and shear stresses, they show 2-3\% larger inner peak for Reynolds streamwise stress.

The terms that significantly contribute to the turbulence production in the current TBLs are $-\overline{u^{\prime} v^{\prime}} \partial U / \partial y$ and $\overline{v^{\prime 2}} \partial U / \partial y$. These terms are present in the equations for $\overline{u^{\prime 2}}$ and $-\overline{u^{\prime} v^{\prime}}$ respectively, in the transport equation for the turbulent stress $\overline{u_{i}^{\prime} u_{j}^{\prime}}$ (Skaare and Krogstad, 1994). The profiles of these terms are shown in Fig. 5 where the inner peaks are located at $y^{+} \approx 12$ and $y^{+} \approx 18$, respectively. The peak heights of these terms in the APG-TBL are roughly $15 \%$ and $30 \%$ larger than in the ZPG-TBL. This shows a larger turbulence production in the APG-TBL as compared to the ZPG-TBL in the buffer layer. No significant outer peaks are found in either of these terms. For the APG-TBL, this is in contrast to the findings of Skaare and Krogstad (1994) who found two distinct peaks in the inner and outer regions for each of these terms. They could not resolve the locations of the inner peaks because of their hot-wire data having low-spatial-resolution in the wall-normal direction. However, their outer peaks were observed at locations where stresses are maximum $(y / \delta=0.45)$. The absence of the significant outer peak in the present study is expected because of the mild APG $(\beta \approx 2)$ as compared to strong APG in Skaare and Krogstad $(1994)(\beta \approx 21)$. In the current measurements, the inner peaks of $-\overline{u^{\prime} v^{\prime}} \partial U / \partial y$ are almost two times stronger than those of $\overline{v^{\prime 2}} \partial U / \partial y$, while both are higher in the APG-TBL than in the ZPG-TBL.

\subsection{POD Modes}

As aforementioned, POD was used to analyse the HSR measurements of the ZPG- and APG-TBLs. As the EuHIT TBLs do not cover the inner-layer, POD was not performed on those data-sets to avoid erroneous measurements. Therefore, the results from here onwards will rely only on the HSR measurements. The distributions of eigenvalues corresponding to the individual POD modes and their cumulative sum as a fraction of the total energy are shown in Fig. 6. The POD modes are sorted conventionally, such that the contribution to TKE decrease with increasing mode number $i$ i.e. $\lambda_{1} \geqslant \lambda_{2} \geqslant \ldots \lambda_{N} \geqslant 0$. The first modes of the ZPG- and APG-TBLs contain $32 \%$ and $42 \%$ of the total energy in the flow, respectively. For $i \geqslant 2$, the relative contribution of each mode is roughly similar between ZPG- and APGTBLs. It is also interesting to observe that more than 100 modes are required to obtain $90 \%$ of the energy which indicates the highly stochastic (random) nature of the TBL.

The contour plots of the streamwise component of the spatial modes $u_{\phi_{i}}^{\prime}$ for $i=\{1,2,3,4,5\}$ are shown in figure 7 and for $i=\{1,11,21,31,41\}$ in figure 8 for both TBLs. As shown from the two figures, the first mode has the largest scales and hence contributes the most to the TKE. With increasing $i$, the scales become smaller and smaller which is the reason for their reduced contribution to the total TKE. The structure of the first two spatial modes looks qualitatively similar to those found in Wu (2014).

Probability density functions (PDFs) of the temporal coefficients corresponding to the first five POD modes for the ZPG- and APG-TBLs are shown in Fig. 9. For each TBL, the first five modes have a similar distribution of their temporal coefficients which appear Gaussian. As all velocity fields (i.e. snapshots) contribute differently to the first and every other POD mode, those fields which have the largest contributions to the large scales of the first mode $\phi_{1}$ can be characterized as the fields with dominant LSMs. The extent of contribution of each velocity field to the first spatial mode is reflected by the magnitude of its temporal coefficient corresponding to $\phi_{1}$. This is an enhanced definition of the basis of the classification described 


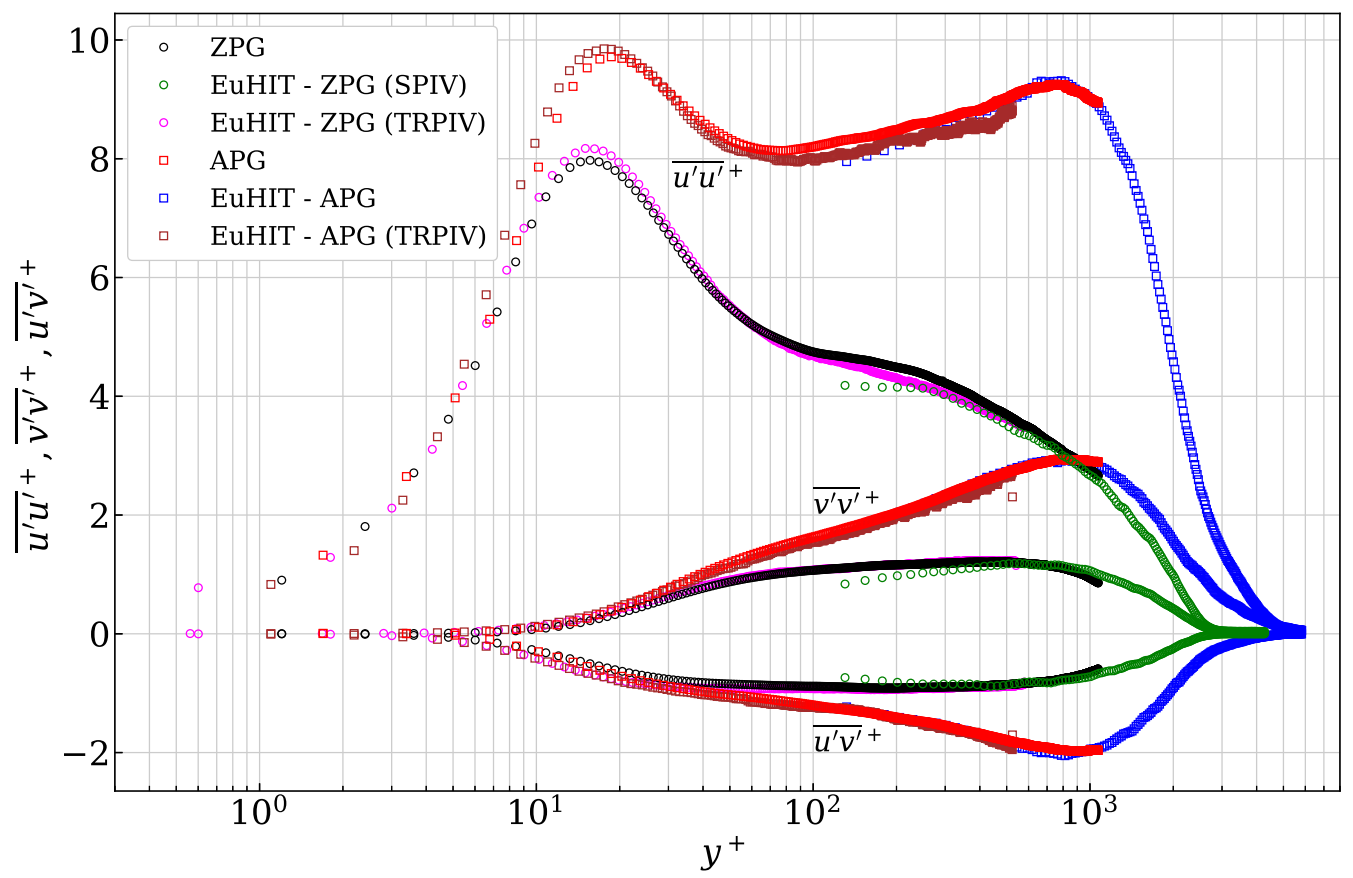

(a)

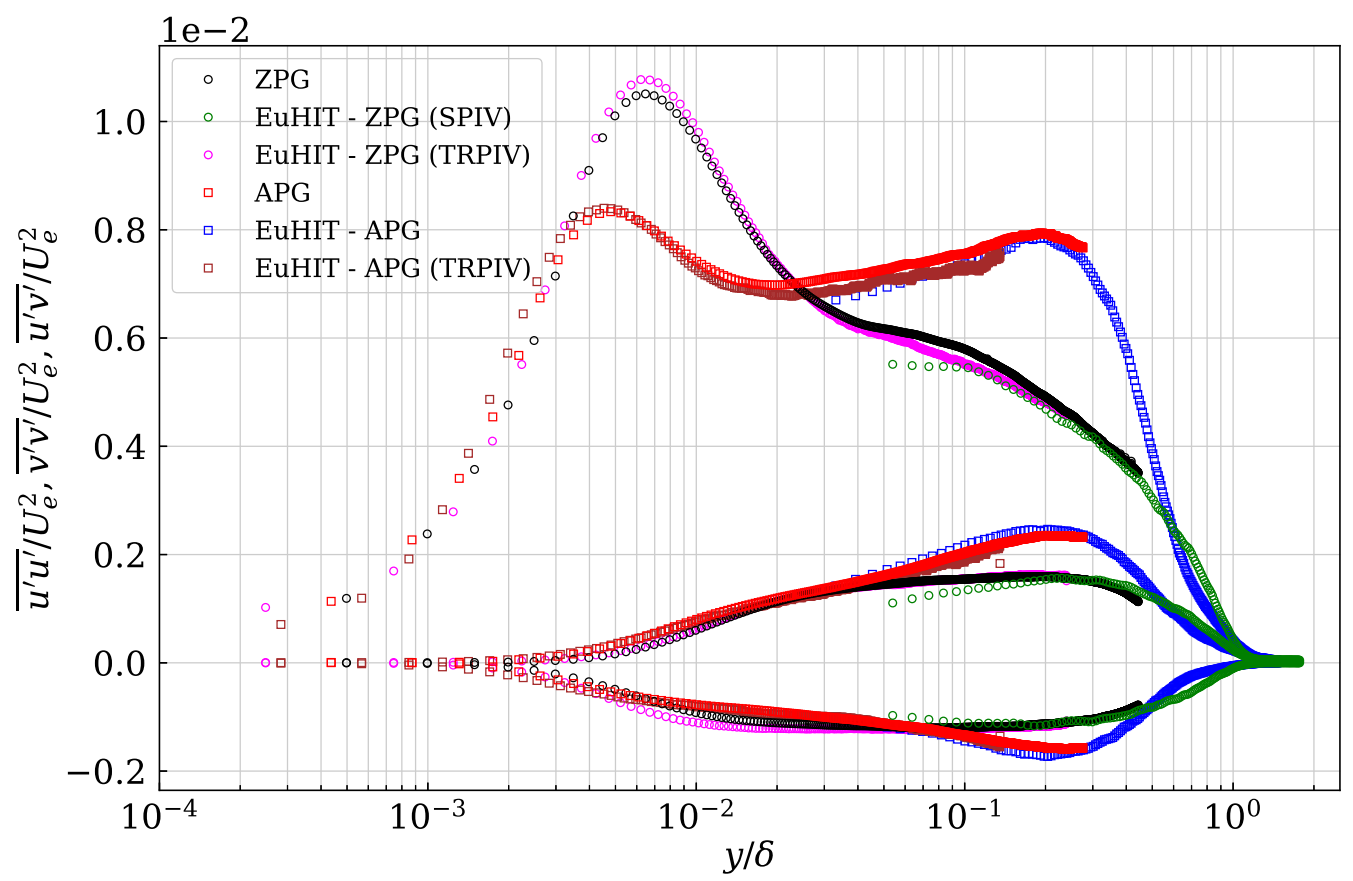

(b)

Figure 4: Reynolds stress profiles scaled in (a) inner variables (b) outer variables. 


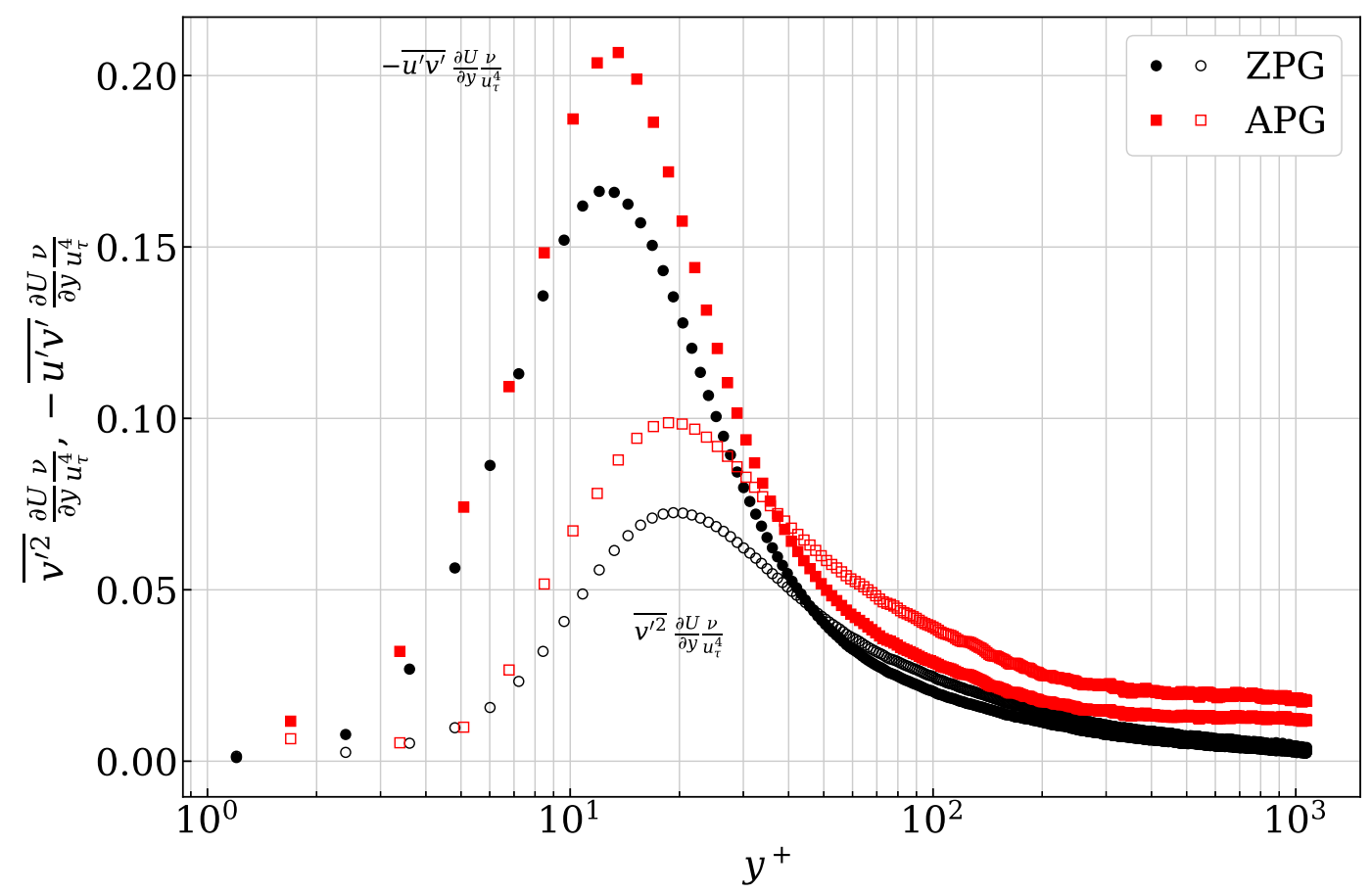

Figure 5: Profiles of dominant terms in turbulence production. 


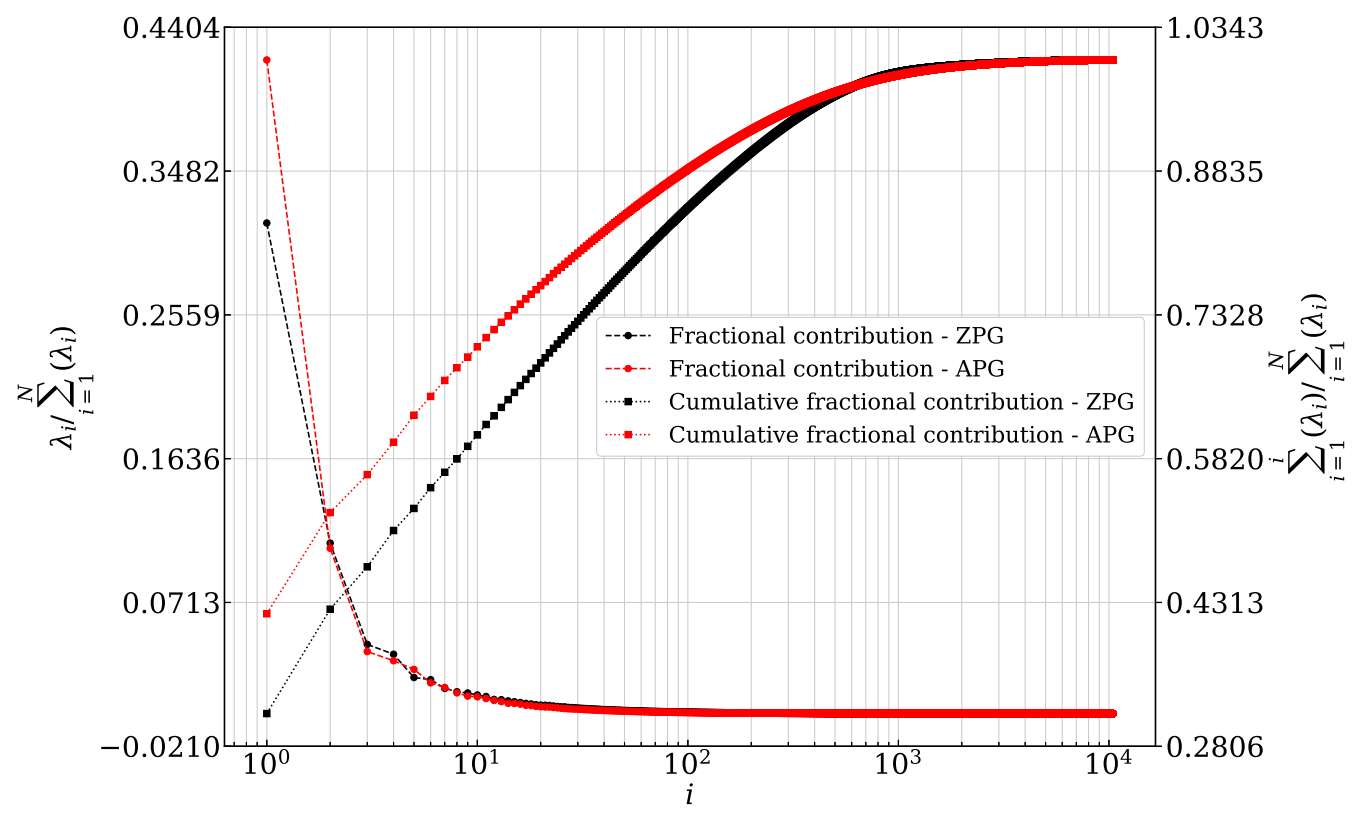

Figure 6: Eigenvalues of individual POD modes and their cumulative sum as a fraction of the total energy of all modes.

in section 2.2. The threshold $K$ in Eq. 10 is selected based on the distribution of $\psi_{1}$. Different values of $K$ would give different extent of the LSMs segmented from the turbulent flow field. Given that the temporal coefficients of the first POD mode have a Euclidean norm of 1 , $K=1$ is chosen as one possible value for the segmentation of the predominant LSMs and their further classification into high and low-momentum events. A sensitivity analysis of the second-order turbulent statistics without LSMs using the classification based on the different values of the threshold factor $K=\{1.0,1.5,2.0\}$ is presented in section 4.4.

To investigate the effect of high- and low-momentum LSMs on turbulent statistics, the flow fields $F$ of each TBL is divided into three parts: (i) high-momentum LSMs $(H)$, (ii) low-momentum LSMs $(L)$ and (iii) SSMs. Further classification of the fields with LSMs into those dominated by high- or low-momentum events is based on the signs of $\psi_{1_{j}}$ and $u_{\phi_{1}}^{\prime}$. As $u_{\phi_{1}}^{\prime}<0$ for the ZPG-TBL, the velocity fields of the ZPG-TBL with high- and low-momentum events are $H^{-}$and $L^{-}$, respectively. In contrast, as $u_{\phi_{1}}^{\prime}>0$ for the APG-TBL, the velocity fields of the APG-TBL with high- and low-momentum events are $H^{+}$and $L^{+}$, respectively. The distributions of the temporal coefficients of velocity fields with LSMs for both TBLs are shown in figure 10 where the red regions correspond to high-momentum events and the blue regions correspond to low-momentum events. These regions are bounded by dotted lines of $\psi_{1_{j}}= \pm \sigma_{\psi_{1}}$ and solid lines of $\operatorname{PDF}\left(\left|\psi_{1_{j}}\right|>\sigma_{\psi_{1}}\right)$. The dashed lines and dash-dotted lines represent $K=1.5$ and $K=2.0$ from figure 10, respectively. 

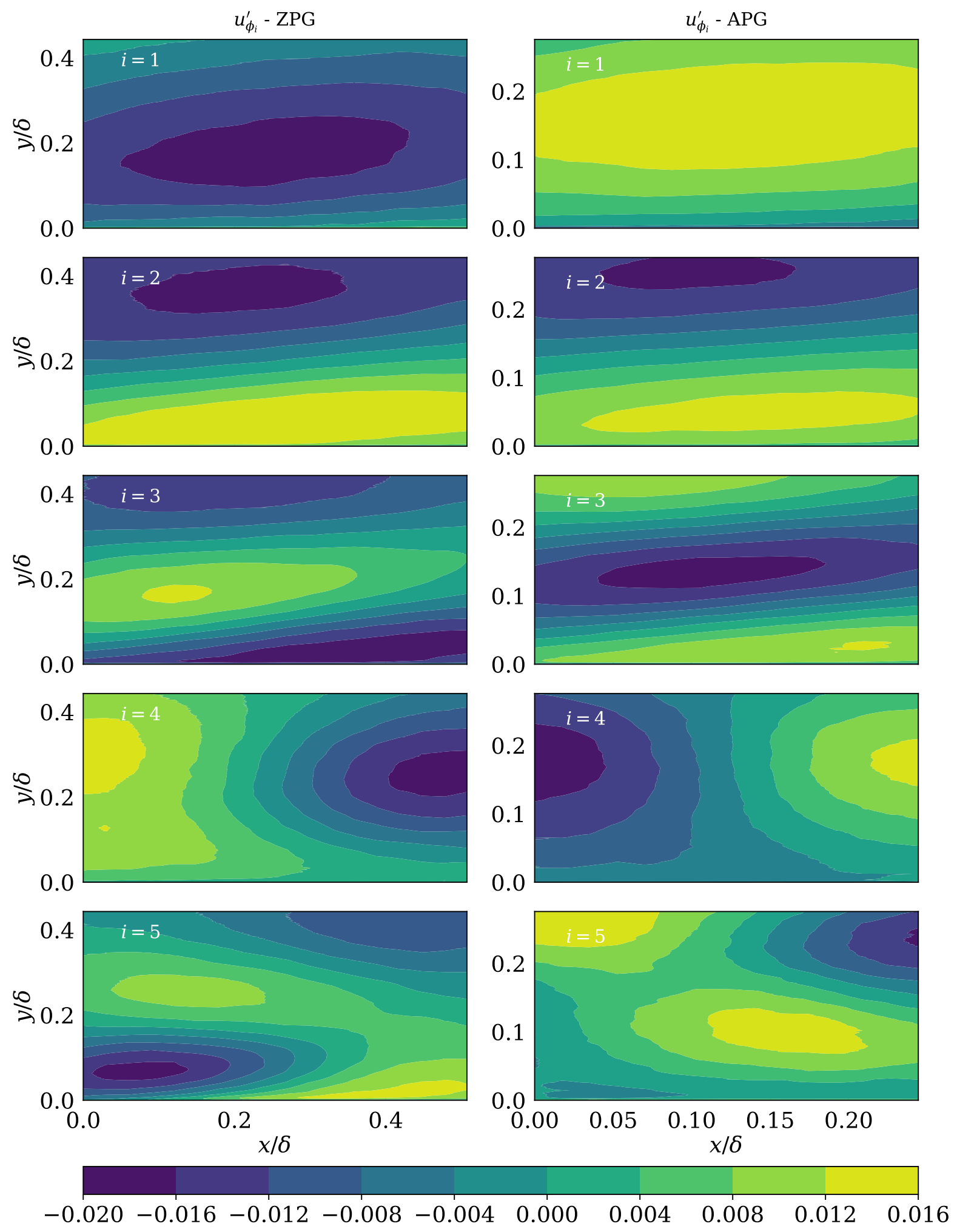

Figure 7: Contour plots of the streamwise components of the first five spatial modes of ZPG-TBL on the left and APG-TBL on the right. 

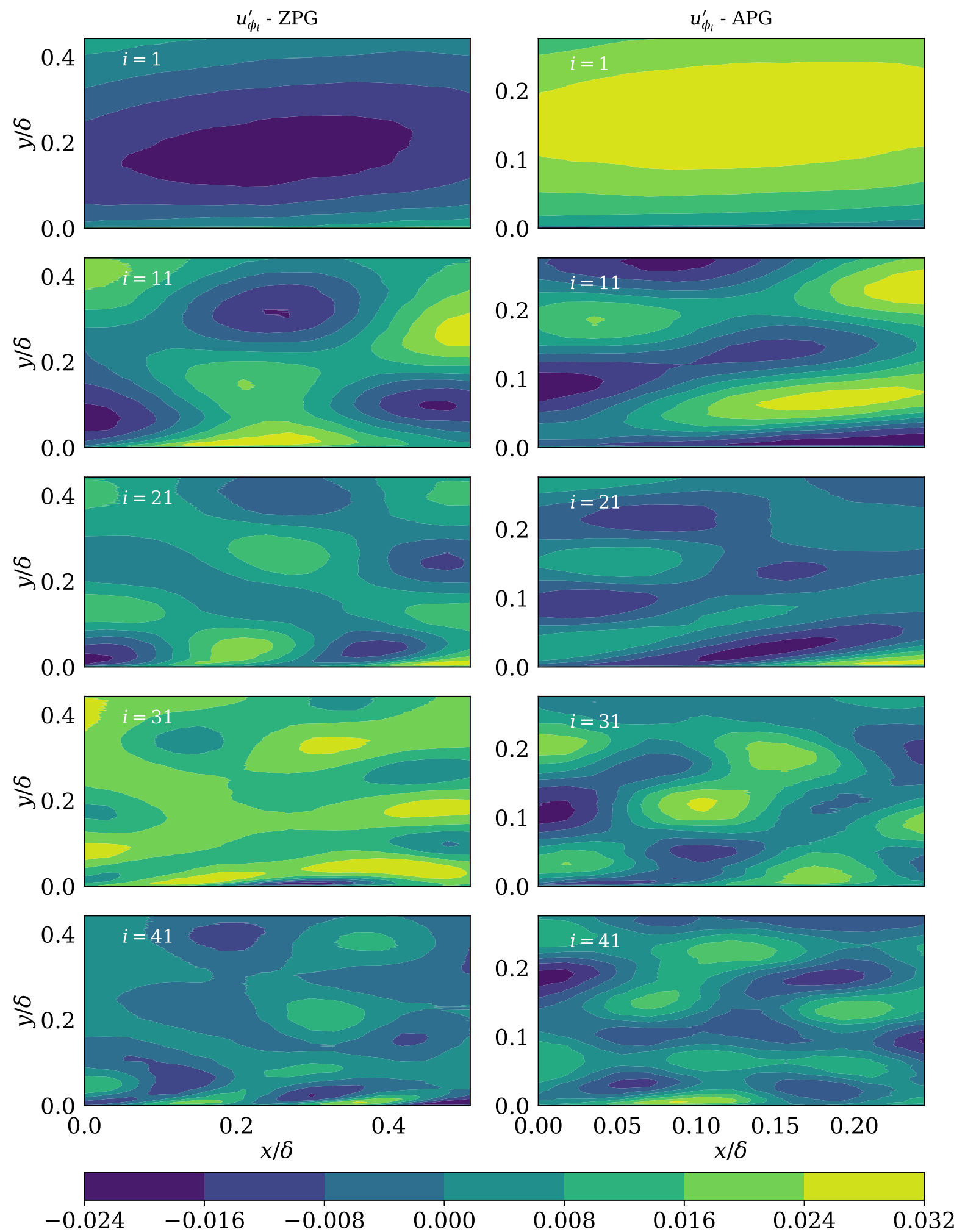

Figure 8: Contour plots of the streamwise components the spatial modes $u_{\phi_{i}}^{\prime}$ of ZPG-TBL on the left and APG-TBL on the right where $i=\{1,11,21,31,41\}$. 

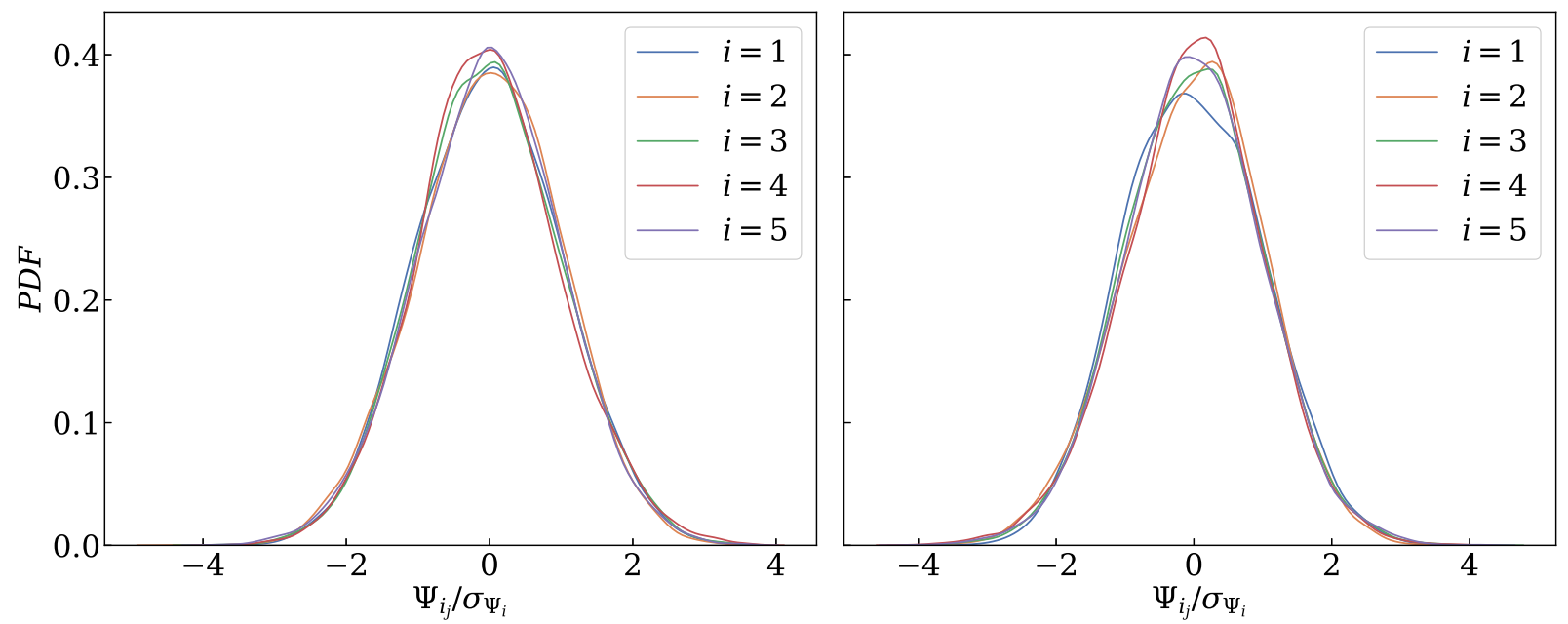

Figure 9: PDFs of temporal coefficients corresponding to the first five most energetic POD modes for (a) ZPG-TBL and (b) APG-TBL. The vertical dotted lines correspond to the $\psi_{1} / \sigma_{\psi_{1}}= \pm 1.0$, dashed lines to $\psi_{1} / \sigma_{\psi_{1}}= \pm 1.5$ and the solid lines to $\psi_{1} / \sigma_{\psi_{1}}= \pm 2.0$.
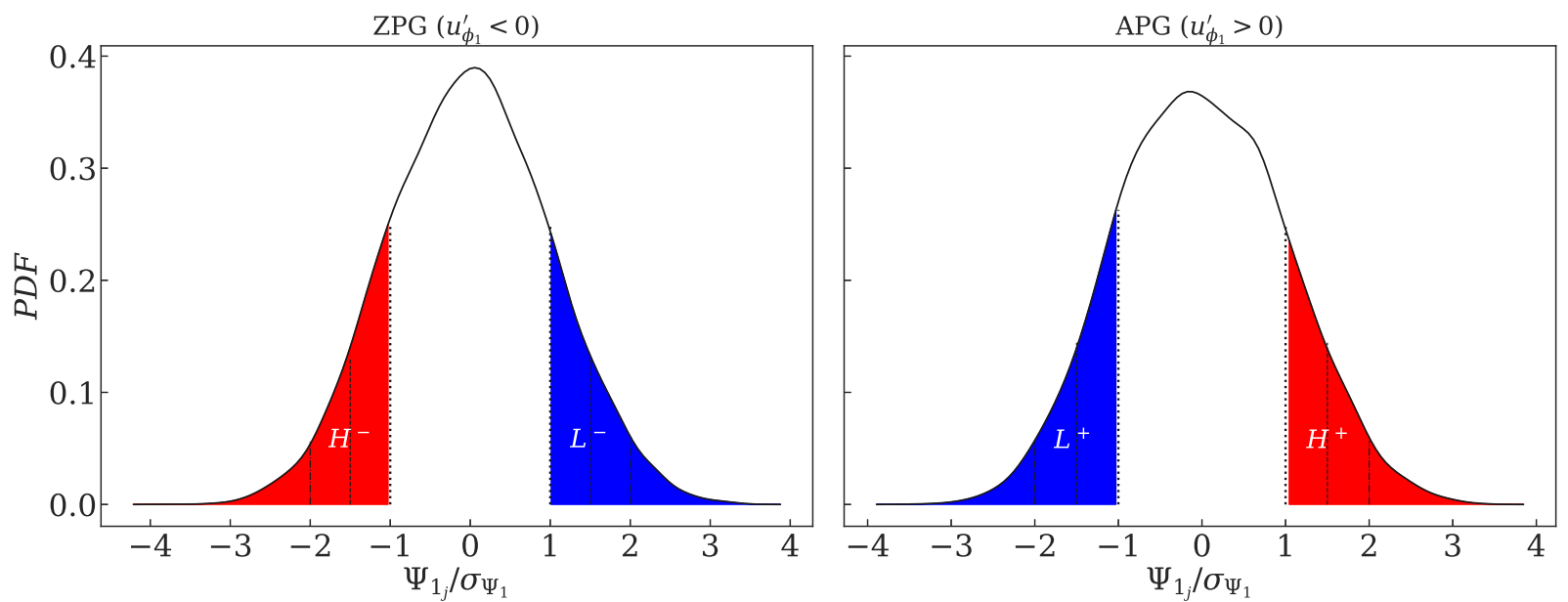

Figure 10: Classification of LSMs in to high- and low-momentum events depicted on the PDFs of temporal coefficients corresponding to the first POD modes. The vertical dotted lines correspond to the $\psi_{1} / \sigma_{\psi_{1}}= \pm 1.0$, dashed lines to $\psi_{1} / \sigma_{\psi_{1}}= \pm 1.5$ and the dash-dotted lines to $\psi_{1} / \sigma_{\psi_{1}}= \pm 2.0$. 


\subsection{Conditional Averaging of the Turbulent Statistics}

The conditional averaging of turbulent statistics is performed on the sub-fields $H$ and $L$ as presented in table 4 . The - and + superscripts differentiate between the nature of the first POD mode of the TBLs (see sections 2.2 and 4.2). The subscripts $h$ and $l$ represent the conditionally averaged statistics from the $H$ and $L$ sub-fields, respectively. This notation will be followed in the profiles of the conditionally averaged turbulent statistics.

Table 4: The nomenclature of the conditionally averaged turbulent statistics.

\begin{tabular}{lll|lll}
\hline \hline \multicolumn{3}{c}{ High-momentum } & \multicolumn{3}{c}{ Low-momentum } \\
Property ZPG & APG & Property ZPG & APG \\
\hline$U_{h}$ & $\overline{u\left[H^{-}\right]}$ & $\overline{u\left[H^{+}\right]}$ & $U_{l}$ & $\overline{u\left[L^{-}\right]}$ & $\overline{u\left[L^{+}\right]}$ \\
$\overline{u^{\prime} u^{\prime}} h_{h}$ & $\overline{\left(u^{\prime}\left[H^{-}\right]\right)^{2}}$ & $\overline{\left(u^{\prime}\left[H^{+}\right]\right)^{2}}$ & $\overline{u^{\prime} u_{l}^{\prime}}$ & $\overline{\left(u^{\prime}\left[L^{-}\right]\right)^{2}}$ & $\overline{\left(u^{\prime}\left[L^{+}\right]\right)^{2}}$ \\
$\overline{v^{\prime} v^{\prime}}{ }_{h}$ & $\overline{\left(v^{\prime}\left[H^{-}\right]\right)^{2}}$ & $\overline{\left(v^{\prime}\left[H^{+}\right]\right)^{2}}$ & $\overline{v^{\prime} v^{\prime}}$ & $\overline{\left(v^{\prime}\left[L^{-}\right]\right)^{2}}$ & $\overline{\left(v^{\prime}\left[L^{+}\right]\right)^{2}}$ \\
$\overline{u^{\prime} v^{\prime}}{ }_{h}$ & $\overline{u^{\prime}\left[H^{-}\right] v^{\prime}\left[H^{-}\right]} \overline{u^{\prime}\left[H^{+}\right] v^{\prime}\left[H^{+}\right]} \overline{u^{\prime} v^{\prime}}{ }_{l}$ & $\overline{u^{\prime}\left[L^{-}\right] v^{\prime}\left[L^{-}\right]}$ & $\overline{u^{\prime}\left[L^{+}\right] v^{\prime}\left[L^{+}\right]}$ \\
\hline
\end{tabular}

The profiles of these statistics, along with the dominant term in turbulence production $-\overline{u^{\prime} v^{\prime}} \frac{\partial U}{\partial y}$ scaled in viscous units are shown in figure 12 for the ZPG- and APG-TBLs. The profiles from the original ensemble have also been included for comparison. As shown in the mean velocity profiles of the ZPG-TBL (figure 12(a)), high-momentum events have a higher mean velocity when compared to the original ensemble, while the opposite is true for the low-momentum events. A similar trend is observed in the APG-TBL (figure 12(b)), but profiles of high- and low-momentum motions are farther from the original ensemble in the outer region when compared to the ZPG-TBL which shows that the activity of the LSMs is enhanced by the APG in the outer layer.

For the Reynolds streamwise stress in the ZPG-TBL (figure 12(c)), the high-momentum events contribute more near the wall and in the log region when compared to the lowmomentum events while the opposite is the case in the wake region. Near the wall, highmomentum events have positive contributions and low-momentum events have negative contributions to Reynolds streamwise stress. This is in agreement with observations of Guemes et al. (2019), who performed similar investigations in a ZPG-TBL. The near-wall peak in $\overline{u^{\prime} u^{\prime}} h$ is about $22 \%$ more pronounced compared to the original ensemble. Both high- and low-momentum events produce an outer peak that is almost as strong as the inner peak in the original ensemble.

Similarly, high-momentum events contribute more to the Reynolds wall-normal stresses than low-momentum from the wall to the end of the log-layer and the opposite is the case in the wake region (see figure 12(e)). The same near-wall distribution is also found in Reynolds shear stress (see figure 12(g)). The Reynolds streamwise and shear stress profiles for highand low-momentum events are up to two times stronger in the wake region compared to 
the corresponding profiles from the original ensembles. This leads to the conclusion that the LSMs have significantly strong activity in the outer region, especially in the streamwise velocity fluctuations. This is also evident from the profiles computed from the fluctuating velocity fields other than those with LSMs $\left(\overline{u_{i}^{\prime} u_{j_{m}}^{\prime}}\right)$, which are below the profiles from the original ensemble in the outer region. Another clear observation in the Reynolds stresses is that the cross-over point, the location in $y^{+}$where the of the high- and low-momentum profiles cross over each other, has conditioned Reynolds stress values higher than the original ensemble which is contradictory to the findings of Guemes et al. (2019). Moreover, Guemes et al. (2019) found that the cross-over points in all Reynolds stresses are at nearly the same wall-normal location, which is not the case in the present study. This is probably because the HSR data in this study does not cover the whole boundary layer, which affects the output of POD and hence the classification such that the cross-over point falls at different $y$ location for different Reynolds stresses.

In the APG-TBL case, the contributions of the high- and low-momentum LSMs to Reynolds stresses (see figure 12-\{(d),(f),(h)\}) show similar trends as in the ZPG-TBL, but their outer peaks are slightly more distant from the original ensemble. This shows the further activation of LSMs in the presence of an APG. Another observable difference is that the cross-over point of the LSM profiles in the APG-TBL moves further from the wall than in the ZPG-TBL.

The profiles of $-\overline{u^{\prime} v^{\prime}} \frac{\partial U}{\partial y}$ show that high- and low-momentum LSMs have inner peaks which are about $25 \%$ stronger and weaker than the original ensemble respectively (see figure 12(i)). The cross-over point in these profiles is also above the original ensemble and is located near the wall-normal location of the cross-over point in the Reynolds shear stress profiles. These changes in the turbulence production near the wall are in agreement with the results of Hutchins and Marusic (2007a), who suggest that the LSMs of the log layer have footprints in the near-wall region. In the APG-TBL (see figure 12(j)), this spread between the highand low-momentum LSMs is about 50\% larger than in the ZPG-TBL, and their cross-over point is also farther in wall-normal direction. This shows that the TKE production due to the high- and low-momentum events is enhanced and reduced, respectively, by the presence of an APG.

\subsection{Sensitivity analysis}

As described in section 2.2, the flow-field is separated into large- and small-scale motions based on a threshold value of the temporal coefficient of the first (i.e. the most energetic) POD mode, $\psi_{1_{j}}$. As $\psi_{1}$ has a unitary Euclidean norm, the threshold factor $K$ is taken as 1. It is, however, worthwhile to investigate the effect of larger values of $K$ (i.e. 1.5 and 2 ) on the conditionally averaged turbulent statistics to find out what portion of LSMs will be subtracted if the larger values of $K$ are used. Because of the Gaussian nature of the distribution of $\psi_{1}$ as shown in figure 9 , fewer and fewer number of velocity fields match the criterion $\left|\psi_{1}\right|>K \sigma_{\psi_{1}}$ for values of $K$ increasing from 1.0 to 2.0. Table 5 lists the percentage values of the number of the velocity fields matching this criterion for $K=\{1.0,1.5,2.0\}$. As evident, the effect of a mild APG on these statistics is negligible as these statistics are very similar for both TBLs.

The sensitivity of Reynolds stresses to the sorted LSMs based on varying the values of $K$ is analysed below. Figure 13 shows the profiles of Reynolds stresses in a ZPG- and an 


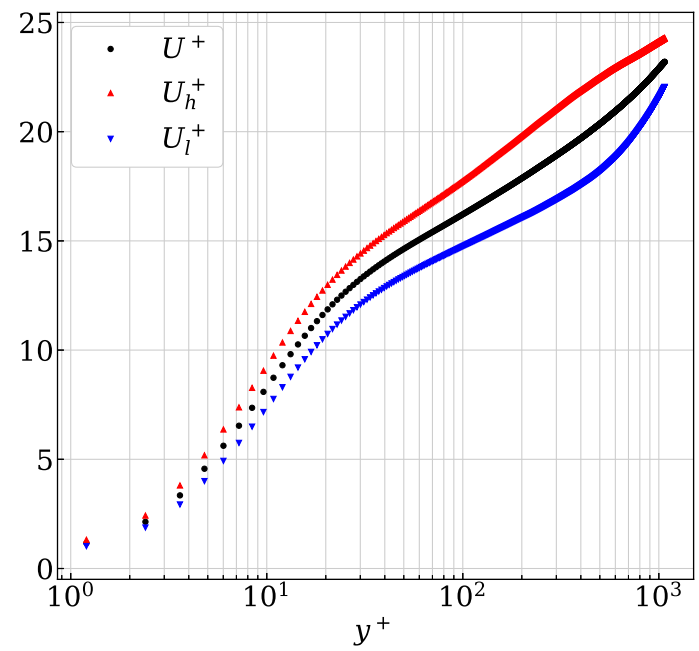

(a)

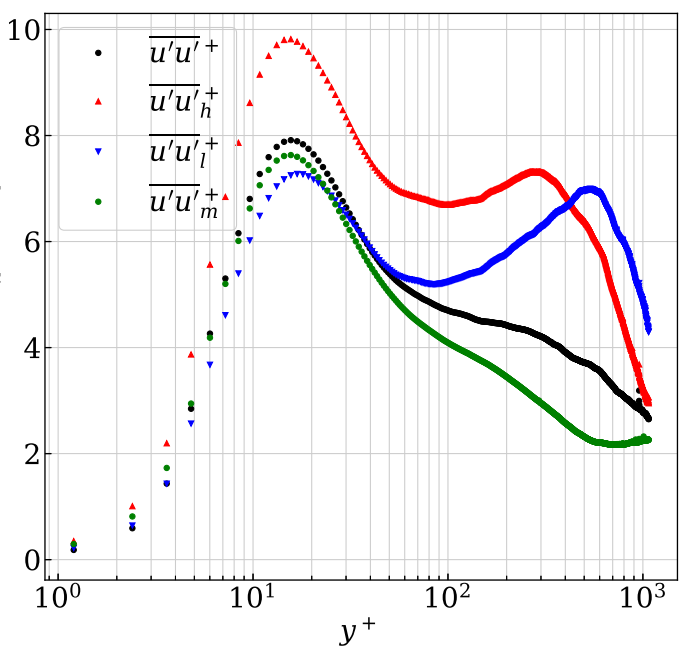

(c)

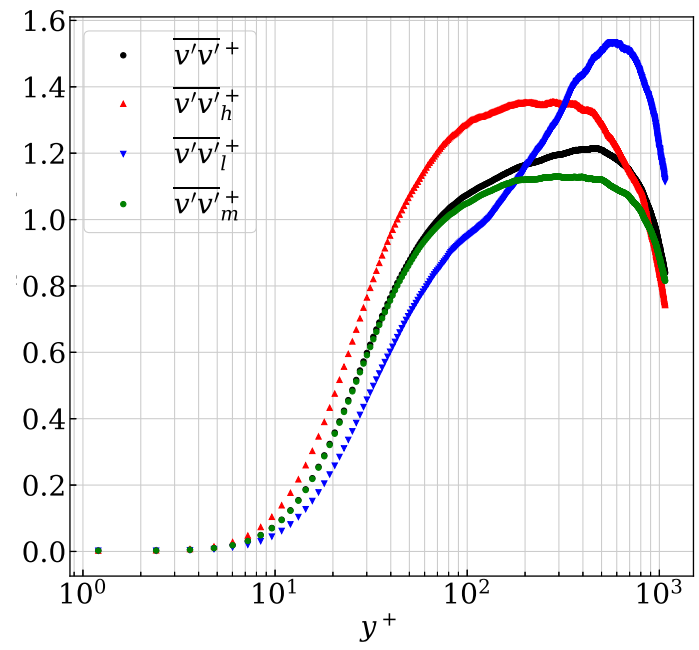

(e)

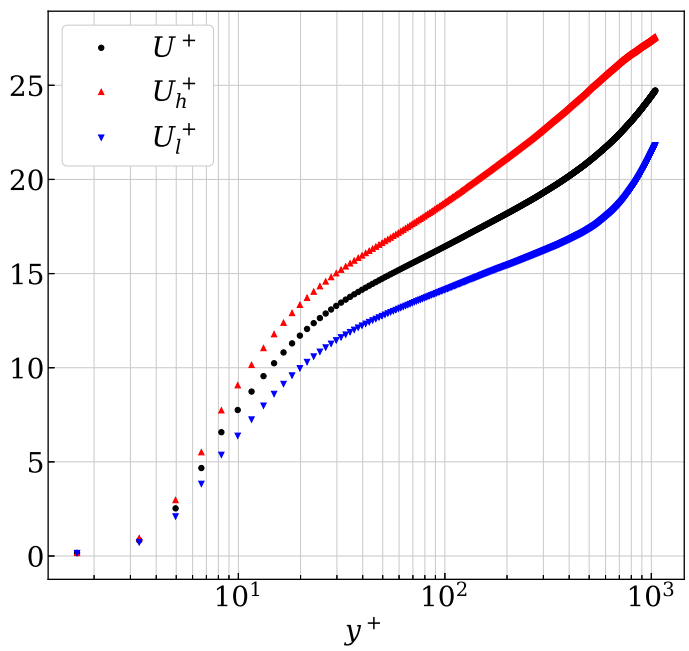

(b)

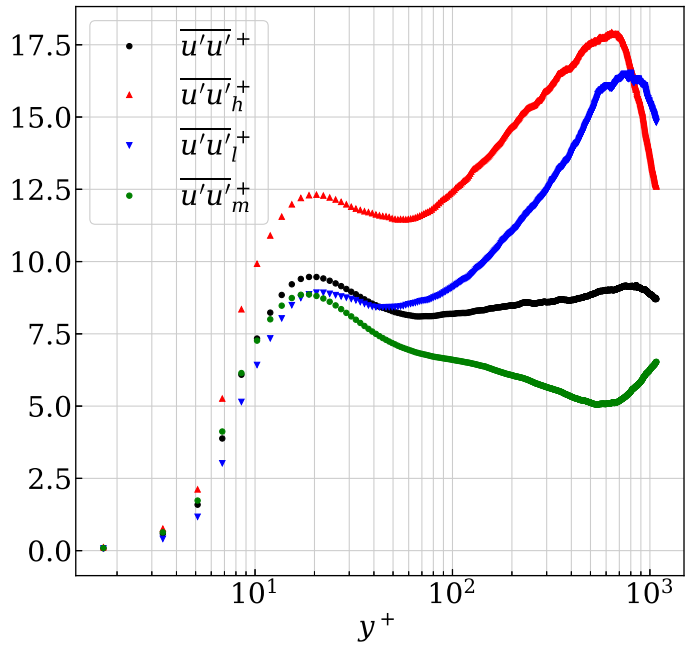

(d)

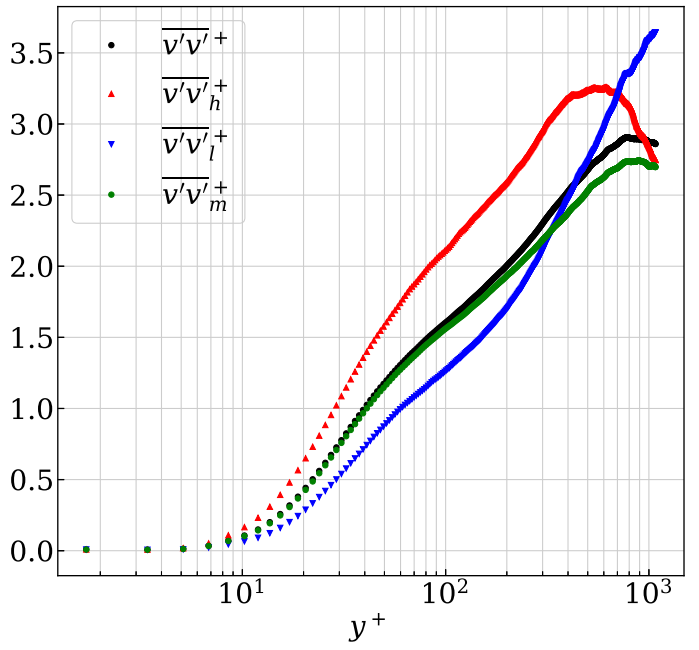

(f)

See caption on the next page. 


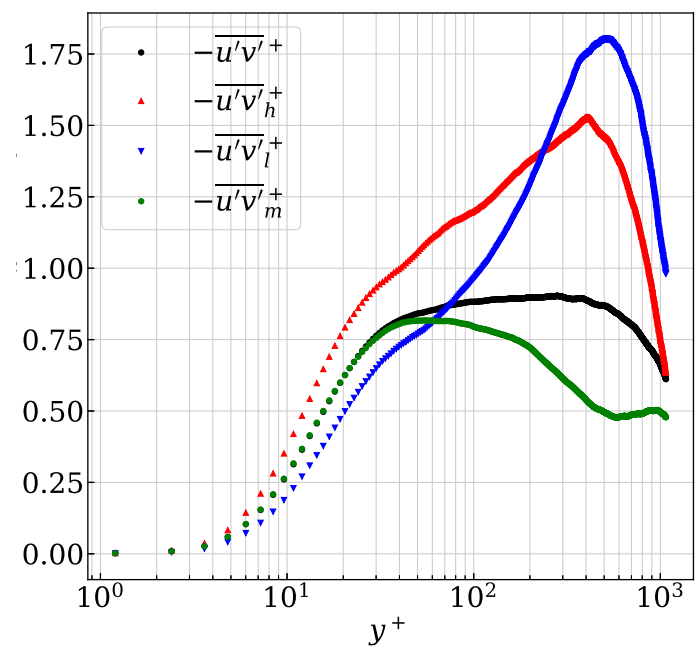

$(\mathrm{g})$

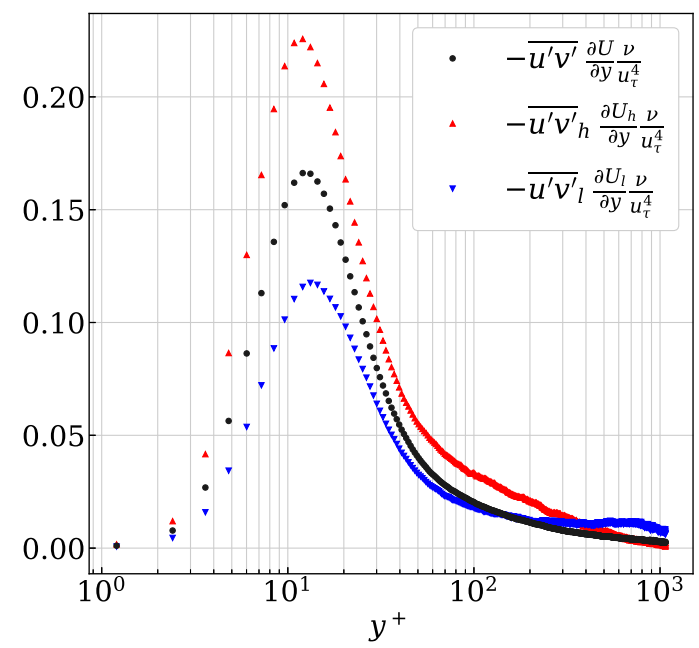

(i)

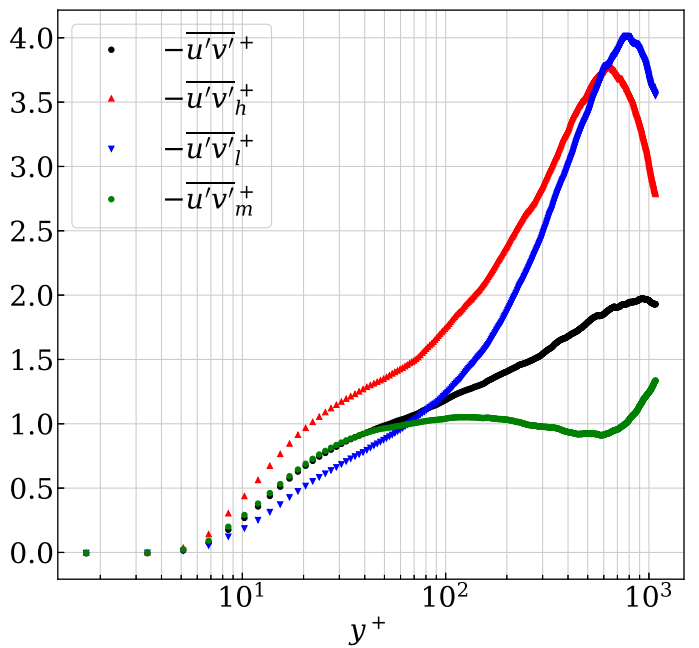

(h)

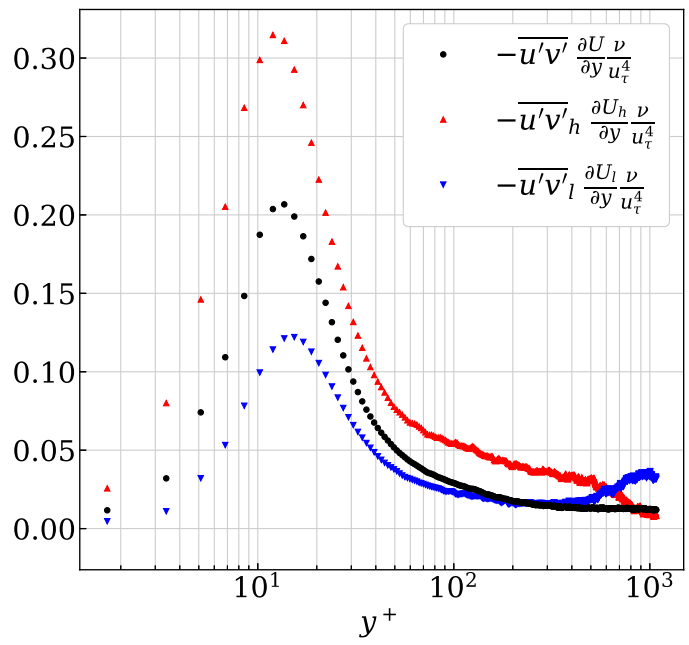

$(\mathrm{j})$

Figure 12: Mean streamwise velocity, Reynolds streamwise, wall-normal and shear stresses and turbulence production term showing the effect of high- and low-momentum LSMs in ZPG-TBL (a),(c),(e),(g),(i), and APG-TBL (b),(d),(f),(h),(j), respectively. The green circles in (c)...(h) show the profiles computed from the fluctuating velocity fields other than those with LSMs. 
Table 5: Percentage of velocity fields with $\left|\psi_{1}\right|>K \sigma_{\psi_{1}}$ for $K=\{1.0,1.5,2.0\}$.

\begin{tabular}{ccc}
\hline \hline & ZPG & APG \\
$K$ & $(\%)$ & $(\%)$ \\
\hline 1.0 & 32.7 & 33.0 \\
1.5 & 13.6 & 13.2 \\
2.0 & 4.2 & 4.0 \\
\hline
\end{tabular}

APG-TBL, computed from ensembles without the fluctuating velocity fields whose $\psi_{1}$ values are larger than $K \sigma_{\psi_{1}}$ where $K=\{1.0,1.5,2.0\}$. These profiles are normalized with the corresponding profiles from the original ensemble. As a larger contribution of the LSMs are removed, the Reynolds stress values are lower than the original ensemble. As shown in figure 13, the effect of a change in $K$ is felt in all of the Reynolds stress profiles in the inner and outer region, but it is more pronounced in the outer region of the Reynolds streamwise and shear stresses. Reynolds wall-normal stresses show very little response to a change in $K$. It is found that the reduction in the Reynolds streamwise stress is the highest, roughly $38 \%$ in the ZPG and $43 \%$ in the APG, for the smallest value of $K$, which is expected as more instantaneous fluctuating velocity fields with larger $\psi_{1}$ are excluded from the ensembles even though they are significant contributors to the TKE. This reduction effect becomes less and less as $K$ increases from 1 to 2 and becomes nearly $10 \%$ in the ZPG-TBL and $12 \%$ in the APG-TBL at $K=2$. Although the reduction of the stresses in the inner region after removing LSMs is minimal, it shows that the near-wall scales are affected by the LSMs of the outer region and this effect is also slightly amplified in a mild APG.

In the Reynolds shear stress, the reduction for different values of $K$ is about $10 \%$ and $15 \%$ larger compared to the Reynolds streamwise stress in the ZPG and APG, respectively. One can conclude that LSMs based on $K=1$ contribute up to $50 \%$ of the Reynolds shear stress in the outer region of the boundary layer. This consistent with the results of Guala et al. (2006) who report that large-scale motions carry over one-half of $-\overline{u^{\prime} v^{\prime}}$.

Profiles of the mean streamwise velocity $U$ and the dominant turbulence production term $-\overline{u^{\prime} v^{\prime}} \frac{\partial U}{\partial y}$ are almost insensitive to the change in $K$ and hence, those have not been presented here for the purpose of brevity.

\section{Conclusion}

POD analysis has been performed on the fluctuating velocities of the flow field of a ZPGand an APG-TBL obtained using high-spatial-resolution PIV. Based on a threshold value $K$, the temporal coefficients of the first POD mode are used to classify the flow field into those with large- and small-scale motions. The fields with large-scale structures are further divided into those with high- and low-momentum events based on the nature of the first POD mode and the values of its temporal coefficients. The conditionally averaged turbulent statistics validate the findings of previous studies in which large-scale motions have significant 


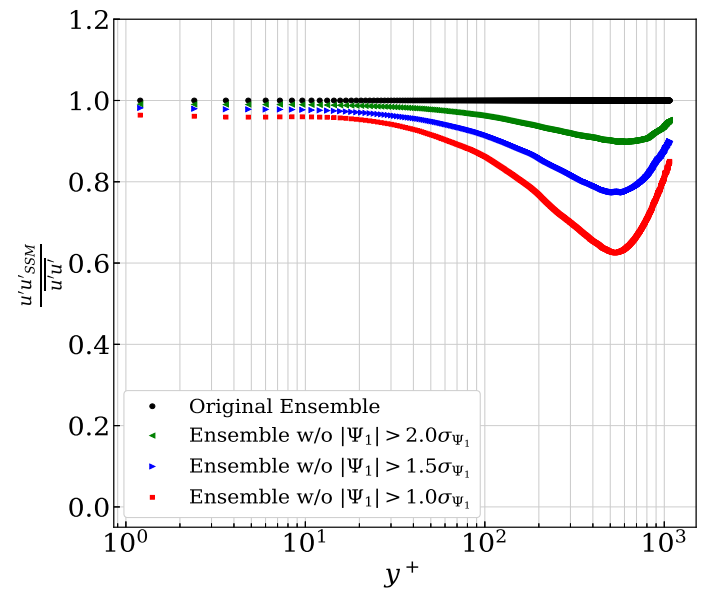

(a)

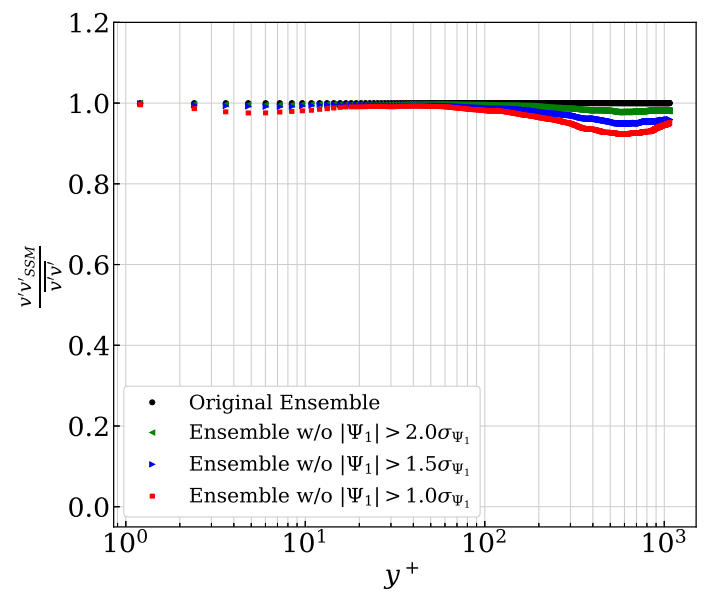

(c)

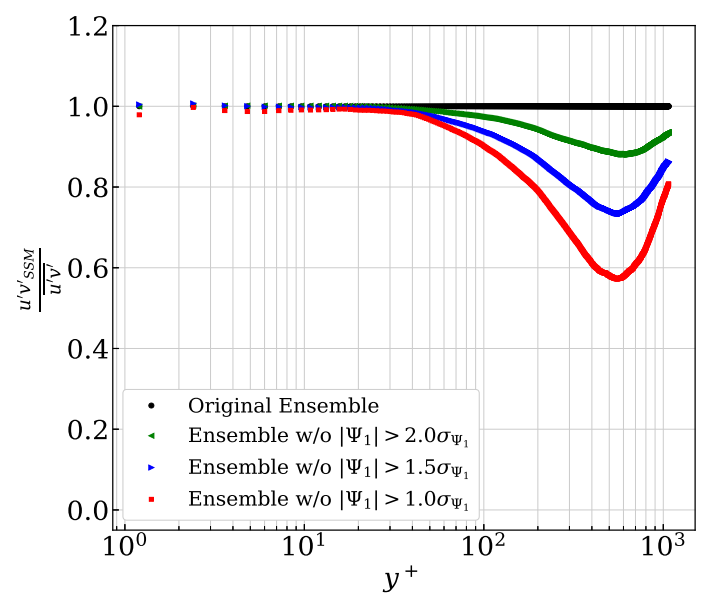

(e)

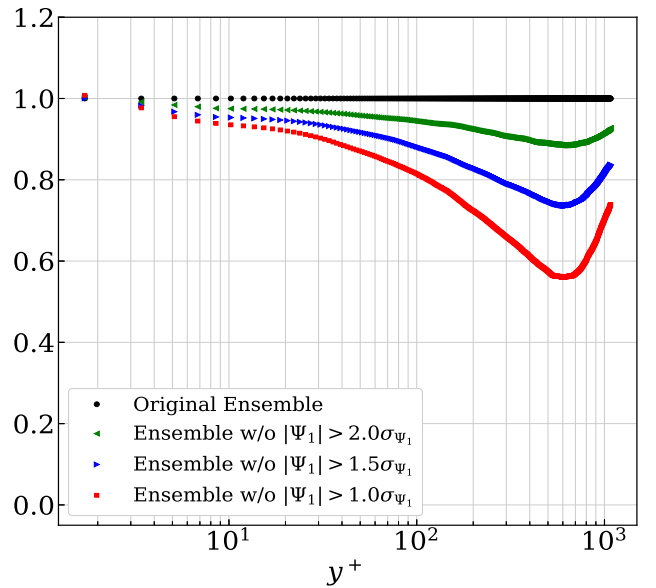

(b)

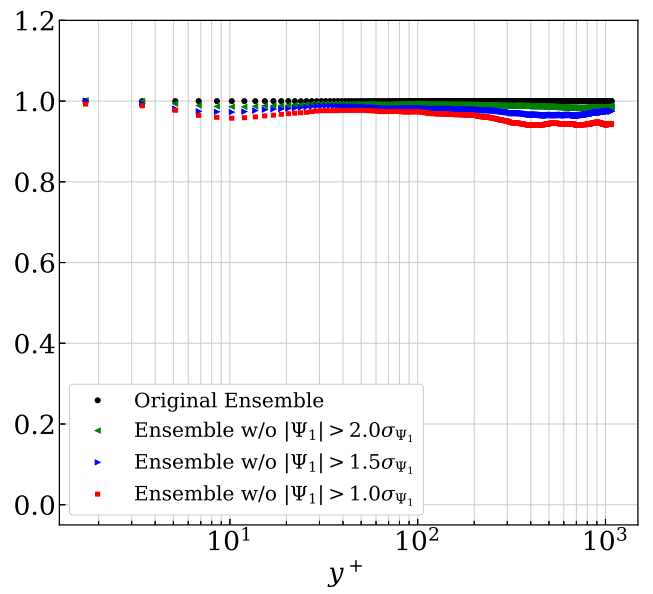

(d)

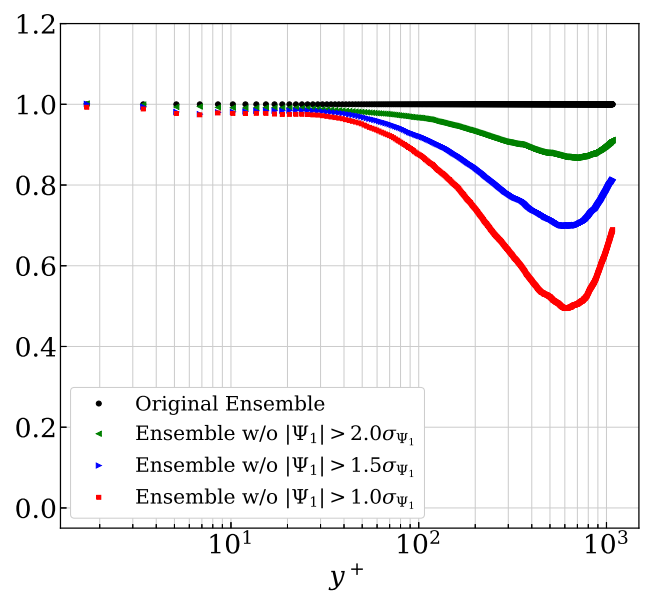

(f)

Figure 13: Reynolds streamwise, wall-normal and shear stresses of in ZPG-TBL (a),(c),(e), and APG-TBL (b),(d),(f), respectively, without the fluctuating velocity fields whose $\psi_{1}$ values are larger than $K \sigma_{\psi_{1}}$ where $K=\{1.0,1.5,2.0\}$. 
contributions in the outer region and contribute largely to Reynolds streamwise and shear stresses in a ZPG-TBL. These motions become even more energized in the presence of a mild APG. In the near-wall region, the high- and low-momentum events contribute more and less, respectively, to the turbulent statistics when compared to the original ensemble. Their effect is reversed in the outer region following a cross-over point in the Reynolds stress profiles, which always happens to be above the values averaged over the original ensemble. The two notable differences in the APG-TBL are: (i) high- and low-momentum events are more energized and (ii) the cross-over point moves farther from the wall in the APG-TBL than in the ZPG-TBL.

Removing the velocity fields with the LSMs based on $K=1$, The estimate of the Reynolds streamwise stress and Reynolds shear stress from the remaining velocity fields in the ZPGTBL are reduced by up to $42 \%$ compared to the original ensemble. This reduction effect is enhanced by roughly $10 \%$ in a TBL with a mild APG. This further signifies the conclusion that the LSMs are amplified in the presence of a mild APG.

\section{Acknowledgements}

The authors would like to acknowledge the support of the Australian Government for this research through an Australian Research Council Discovery grant. C. Atkinson was supported by the ARC Discovery Early Career Researcher Award (DECRA) fellowship. The research was also benefited from computational resources provided by the Pawsey Supercomputing Centre and through the NCMAS, supported by the Australian Government. The computational facilities supporting this project included the NCI Facility, the partner share of the NCI facility provided by Monash University through an ARC LIEF grant and the Multi-modal Australian ScienceS Imaging and Visualisation Environment (MASSIVE).

Muhammad Shehzad acknowledges the Punjab Educational Endowment Fund (PEEF), Punjab, Pakistan for funding his PhD research. Bihai Sun and Daniel Jovic gratefully acknowledge the support through an Australian Government Research Training Program (RTP) Scholarship.

\section{References}

Bakewell Jr, H.P., Lumley, J.L., 1967. Viscous sublayer and adjacent wall region in turbulent pipe flow. The Physics of Fluids 10, 1880-1889.

Barros, J.M., Christensen, K.T., 2019. Characteristics of large-scale and superstructure motions in a turbulent boundary layer overlying complex roughness. Journal of Turbulence 20, $147-173$.

Bourassa, C., Thomas, F., 2009. An experimental investigation of a highly accelerated turbulent boundary layer. Journal of Fluid Mechanics 634, 359-404.

Bross, M., Eich, F., Schanz, D., Novara, M., Schröder, A., Kähler, C., 2021. Superstructures in turbulent boundary layers with pressure gradients. PAMM 20, e202000257.

Bross, M., Fuchs, T., Kähler, C.J., 2019. Interaction of coherent flow structures in adverse pressure gradient turbulent boundary layers. Journal of Fluid Mechanics 873, 287-321. 
Cuvier, C., Srinath, S., Stanislas, M., Foucaut, J., Laval, J., Kähler, C., Hain, R., Scharnowski, S., Schröder, A., Geisler, R., et al., 2017. Extensive characterisation of a high Reynolds number decelerating boundary layer using advanced optical metrology. Journal of Turbulence 18, 929-972.

Del Alamo, J.C., Jiménez, J., 2003. Spectra of the very large anisotropic scales in turbulent channels. Physics of Fluids 15, L41-L44.

Drozdz, A., Elsner, W., 2011. Detection of coherent structures in a turbulent boundary layer with zero, favourable and adverse pressure gradients, in: Journal of Physics: Conference Series, IOP Publishing. p. 062007.

Eich, F., Kähler, C.J., 2020. Large-scale coherent motions in turbulent boundary layers under an adverse pressure gradient up to flow separation. International Journal of Heat and Fluid Flow 85, 108645.

Fernholz, H., Warnack, D., 1998. The effects of a favourable pressure gradient and of the Reynolds number on an incompressible axisymmetric turbulent boundary layer. part 1. the turbulent boundary layer. Journal of Fluid Mechanics 359, 329-356.

Ganapathisubramani, B., Hutchins, N., Hambleton, W., Longmire, E.K., Marusic, I., 2005. Investigation of large-scale coherence in a turbulent boundary layer using two-point correlations .

de Giovanetti, M., Sung, H.J., Hwang, Y., 2017. Streak instability in turbulent channel flow: the seeding mechanism of large-scale motions. arXiv preprint arXiv:1709.06782 .

Grant, H., 1958. The large eddies of turbulent motion. Journal of Fluid Mechanics 4, 149-190.

Guala, M., Hommema, S., Adrian, R., 2006. Large-scale and very-large-scale motions in turbulent pipe flow. Journal of Fluid Mechanics 554, 521.

Guemes, A., Ianiro, A., Discetti, S., 2019. Experimental assessment of large-scale motions in turbulent boundary layers, in: 13th International Symposium on Particle Image Velocimetry.

Hain, R., Scharnowski, S., Reuther, N., Kahler, C.J., Schroder, A., Geisler, R., Agocs, J., Rose, A., Novara, M., Stanislas, M., et al., 2016. Coherent large scale structures in adverse pressure gradient turbulent boundary layers, in: International Symposium on the Application of Laser and Imaging Techniques to Fluid Mechanics 2016, Springer-Verlag London Ltd.

Hambleton, W., Hutchins, N., Marusic, I., 2006. Simultaneous orthogonal-plane particle image velocimetry measurements in a turbulent boundary layer .

Harun, Z., Monty, J.P., Mathis, R., Marusic, I., 2013. Pressure gradient effects on the largescale structure of turbulent boundary layers. Journal of Fluid Mechanics 715, 477-498.

Hellstrom, L.H., Ganapathisubramani, B., Smits, A.J., 2015. The evolution of large-scale motions in turbulent pipe flow. Journal of Fluid Mechanics , 1-14. 
Hellström, L.H., Sinha, A., Smits, A.J., 2011. Visualizing the very-large-scale motions in turbulent pipe flow. Physics of Fluids 23, 011703.

Houra, T., Tsuji, T., Nagano, Y., 2000. Effects of adverse pressure gradient on quasi-coherent structures in turbulent boundary layer. International journal of heat and fluid flow 21, 304311.

Hutchins, N., Marusic, I., 2007a. Evidence of very long meandering features in the logarithmic region of turbulent boundary layers. Journal of Fluid Mechanics 579, 1-28.

Hutchins, N., Marusic, I., 2007b. Large-scale influences in near-wall turbulence. Philosophical Transactions of the Royal Society A: Mathematical, Physical and Engineering Sciences 365, 647-664.

Kitsios, V., Sekimoto, A., Atkinson, C., Sillero, J.A., Borrell, G., Gungor, A.G., Jiménez, J., Soria, J., 2017. Direct numerical simulation of a self-similar adverse pressure gradient turbulent boundary layer at the verge of separation. Journal of Fluid Mechanics 829, 392-419.

Kline, S.J., Reynolds, W.C., Schraub, F., Runstadler, P., 1967. The structure of turbulent boundary layers. Journal of Fluid Mechanics 30, 741-773.

Krogstad, P.Å., Skåre, P.E., 1995. Influence of a strong adverse pressure gradient on the turbulent structure in a boundary layer. Physics of Fluids 7, 2014-2024.

Lee, J., Lee, J.H., Choi, J.I., Sung, H.J., 2014. Spatial organization of large-and very-largescale motions in a turbulent channel flow. Journal of fluid mechanics 749, 818.

Lee, J.H., Sung, H.J., 2009. Structures in turbulent boundary layers subjected to adverse pressure gradients. Journal of Fluid Mechanics 639, 101-131.

Lian, Q.X., 1990. A visual study of the coherent structure of the turbulent boundary layer in flow with adverse pressure gradient. Journal of Fluid Mechanics 215, 101-124.

Liu, Z., Adrian, R., Hanratty, T., 2001. Large-scale modes of turbulent channel flow: transport and structure. Journal of Fluid Mechanics 448, 53.

Lumley, J.L., 1967. The structure of inhomogeneous turbulent flows. Atmospheric turbulence and radio wave propagation .

Mathis, R., Hutchins, N., Marusic, I., 2009. Large-scale amplitude modulation of the smallscale structures in turbulent boundary layers .

Mejia-Alvarez, R., Wu, Y., Christensen, K., 2014. Observations of meandering superstructures in the roughness sublayer of a turbulent boundary layer. International journal of heat and fluid flow 48, 43-51.

Na, Y., Moin, P., 1998. Direct numerical simulation of a separated turbulent boundary layer. Journal of Fluid Mechanics 374, 379-405. 
Nagano, Y., Tagawa, M., Tsuji, T., 1993. Effects of adverse pressure gradients on mean flows and turbulence statistics in a boundary layer, in: Turbulent Shear Flows 8. Springer, pp. $7-21$.

Paakkari, V., et al., 2018. Uniform momentum zones in a turbulent boundary layer above a surface with spanwise traveling wave .

Sekimoto, A., Kitsios, V., Atkinson, C., Jimenez, J., Soria, J., 2017. Intense focal and Reynolds stress structures of a self-similar adverse pressure gradient turbulent boundary layer, in: TSFP DIGITAL LIBRARY ONLINE, Begel House Inc.

Sekimoto, A., Kitsios, V., Atkinson, C., Soria, J., 2019. Outer scaling of self-similar adversepressure-gradient turbulent boundary layers. arXiv preprint arXiv:1912.05143 .

Senthil, S., Atkinson, C., Soria, J., 2020. Analysis of the spanwise extent and time persistence of uniform momentum zones in zero pressure gradient and adverse pressure gradient turbulent boundary layers, in: Journal of Physics: Conference Series, IOP Publishing. p. 012013.

Sirovich, L., 1987. Turbulence and the dynamics of coherent structures. parts i-iii. Quarterly of applied mathematics XLV.

Skaare, P.E., Krogstad, P.å., 1994. A turbulent equilibrium boundary layer near separation. Journal of Fluid Mechanics 272, 319-348.

Soria, J., 1996. An investigation of the near wake of a circular cylinder using a video-based digital cross-correlation particle image velocimetry technique. Experimental Thermal and Fluid Science 12, 221-233.

Spalart, P.R., Watmuff, J.H., 1993. Experimental and numerical study of a turbulent boundary layer with pressure gradients. Journal of Fluid Mechanics 249, 337-371.

Sun, B., Shehzad, M., Jovic, D., Cuvier, C., Willert, C., Ostovan, Y., Foucaut, J.M., Atkinson, C., Soria, J., 2021. Distortion correction of two-component - twodimensional PIV using a large imaging sensor with application to measurements of a turbulent boundary layer flow at $R e_{\tau}=2386$. Under review Experiments in Fluids arXiv:arXiv:2103.00115[physics.flu-dyn].

Taira, K., Brunton, S.L., Dawson, S.T., Rowley, C.W., Colonius, T., McKeon, B.J., Schmidt, O.T., Gordeyev, S., Theofilis, V., Ukeiley, L.S., 2017. Modal analysis of fluid flows: An overview. Aiaa Journal , 4013-4041.

Townsend, A., 1958. The turbulent boundary layer, in: Grenzschichtforschung/Boundary Layer Research. Springer, pp. 1-15.

Wallace, J.M., Eckelmann, H., Brodkey, R.S., 1972. The wall region in turbulent shear flow. Journal of Fluid Mechanics 54, 39-48.

Wark, C.E., Nagib, H., 1991. Experimental investigation of coherent structures in turbulent boundary layers. Journal of Fluid Mechanics 230, 183-208. 
Willert, C., 1997. Stereoscopic digital particle image velocimetry for application in wind tunnel flows. Measurement science and technology 8, 1465.

Willert, C.E., Cuvier, C., Foucaut, J.M., Klinner, J., Stanislas, M., Laval, J.P., Srinath, S., Soria, J., Amili, O., Atkinson, C., et al., 2018. Experimental evidence of near-wall reverse flow events in a zero pressure gradient turbulent boundary layer. Experimental Thermal and Fluid Science 91, 320-328.

Wu, Y., 2014. A study of energetic large-scale structures in turbulent boundary layer. Physics of Fluids 26, 045113.

Zhou, H., Lu, L., 1997. Effect of pressure gradient on coherent structures in a turbulent boundary layer. Science in China Series A: Mathematics 40, 441-448. 